Revue des patrimoines

$26 \mid 2015$

La reconversion des sites et des bâtiments industriels

\title{
Les peintures de la cathédrale Saint-Pierre de Nantes
}

Pierre Curie

\section{OpenEdition}

\section{Journals}

Édition électronique

URL : http://journals.openedition.org/insitu/11856

DOI : 10.4000/insitu. 11856

ISSN : 1630-7305

Éditeur

Ministère de la Culture

Référence électronique

Pierre Curie, «Les peintures de la cathédrale Saint-Pierre de Nantes », In Situ [En ligne], 26 | 2015, mis en ligne le 07 juillet 2015, consulté le 25 mars 2020. URL : http://journals.openedition.org/insitu/11856 ; DOI : https://doi.org/10.4000/insitu. 11856

Ce document a été généré automatiquement le 25 mars 2020

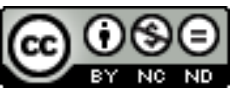

In Situ Revues des patrimoines est mis à disposition selon les termes de la licence Creative Commons Attribution - Pas d'Utilisation Commerciale - Pas de Modification 4.0 International. 


\title{
Les peintures de la cathédrale Saint- Pierre de Nantes
}

\author{
Pierre Curie
}

1 Cette étude a été conduite et ce texte rédigé en 1990-1991 dans le contexte de la préparation d'un Cahier du patrimoine consacré à la cathédrale de Nantes, projet de publication de l'Inventaire général dans une collection nationale qui n'a malheureusement jamais vu le jour.

2 En 2013, à la suite d'une erreur d'appréciation sur le droit d'auteur et de ce que le droit français nomme une contrefaçon, ce texte a paru, tronqué et sous une signature apocryphe, dans un ouvrage publié chez un éditeur privé.

3 In Situ présente ici ce texte dans sa forme originale, in extenso, et sous le nom de son auteur.

\section{Les peintures des $\mathrm{XVII}{ }^{e}$ et $\mathrm{XVIII}{ }^{\mathrm{e}}$ siècles}

4 Depuis quelques années, la peinture ancienne des régions de France attire l'attention des chercheurs et du public. Un tel regain d'intérêt ne s'était plus manifesté depuis les travaux érudits du siècle dernier et il aura fallu la redécouverte de quelques grands oubliés, comme Georges de La Tour, pour que soit de nouveau exploré, le terme n'est pas trop fort, le legs des provinces à l'histoire de l'art français. Du fait des destructions et d'une indifférence plus cruelle encore, la région nantaise ne semble pas pouvoir offrir au curieux ou au savant la possibilité d'une reconstitution de l'activité des peintres et des ateliers sous l'Ancien Régime. Trop d'éléments ont disparu pour qu'on puisse jamais réunir suffisamment d'œuvres et proposer une image cohérente de cette production.

5 Mais nous souhaitons nous tromper, et les quatre tableaux antérieurs à la Révolution que conserve Saint-Pierre sont un démenti partiel à ce constat pessimiste. Miraculés des iconoclasmes, des conflits, mais aussi des changements de goûts, ils illustrent par leur qualité la splendeur et le faste qu'exigeait alors le culte catholique. À Nantes, hélas, 
le sort s'est aussi acharné sur les témoins indirects de cet éclat perdu. Avec l'incendie des archives du chapitre lors des bombardements de la dernière guerre, les chances de trouver des informations susceptibles de renouveler les publications d'avant 1940 se sont considérablement réduites ${ }^{1}$.

6 À la cathédrale, rien ne subsiste des peintures des $\mathrm{XV}^{\mathrm{e}}$ et $\mathrm{XVI}^{\mathrm{e}}$ siècles et seuls quelques fragments de connaissances fort lacunaires nous renseignent sur celles du XVII siècle. Dans la chapelle Notre-Dame-de-Pitié, le beau retable de Tugal Caris (1656) encadrait autrefois une Crucifixion que les érudits locaux attribuaient généralement à Charles Le Brun (1619-1690) ${ }^{2}$ et qu'on croyait disparue lors de la dernière guerre. Récemment retrouvée, l'œuvre restaurée s'est révélée d'une indéniable valeur. À une époque indéterminée, le tableau a été agrandi de deux bandes latérales pour l'adapter au retable; son dépôt dans la crypte date sûrement de $1944^{3}$. Dans les années soixante, on l'avait remplacé par une Apparition de la Vierge à saint Philippe Néri, bonne copie ancienne d'un tableau de Carlo Maratti (1625-1713) ${ }^{4}$ prêtée à la cathédrale par le musée des Beaux-Arts ${ }^{5}$. Reflet du classicisme romain ou bolonais de la première moitié du XVII ${ }^{e}$ siècle, cette toile un peu fade ne présente pas l'intérêt de la Crucifixion retrouvée qui vient de réintégrer son ancien emplacement. Cette belle peinture de la fin du XVII siècle sort peut-être de l'atelier de Charles de La Fosse (1636-1716) et plusieurs autres versions, avec des variantes, en sont connues ${ }^{6}$. S'il s'avérait que l'œuvre se trouvait à Saint-Pierre au XVII ${ }^{\mathrm{e}}$ siècle, on aurait alors la confirmation de la qualité des tableaux de la cathédrale pour cette période, comme le laissait déjà supposer la Remise des clefs à saint Pierre de l'ancien maître-autel due à Charles Errard (fig. 1).

Figure 1

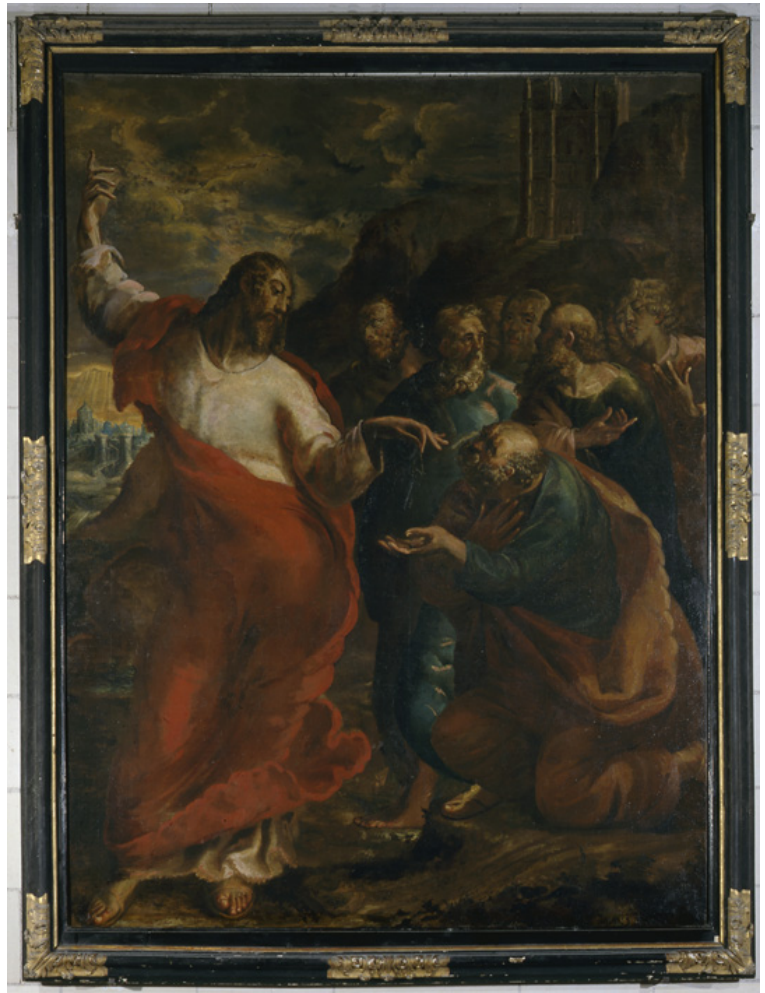

Charles Errard le père. La Remise des clefs à saint Pierre. Nantes, cathédrale Saint-Pierre. Phot. D. Pillet. (c) Région Pays de la Loire - Inventaire général, 8544 226VA. 
7 L'artiste naquit en 1570 à Bressuire, en Poitou ${ }^{7}$. Établi à Nantes en 1599, après la soumission du duc de Mercœur, il dut abjurer ses convictions protestantes en 1615 pour accéder à la très officielle fonction d'architecte des fortifications de Bretagne, poste que l'année précédente Louis XIII, de passage à Nantes, avait souhaité lui attribuer. À la fin de 1618, la fabrique de la cathédrale passa avec Errard un marché de deux mille quatre cents livres concernant l'embellissement du " grand autel et sacrayre du chœur» de Saint-Pierre et la commande du tableau de La Remise des clefs. Celui-ci dut être achevé au cours de l'année suivante mais malheureusement, la date qu'il portait fut effacée par un « restaurateur » du siècle dernier. La composition surprend par sa mobilité dansante et sa grâce toute maniériste. Le geste " précieux et faux ${ }^{8}$ » du Christ s'y oppose, dans une atmosphère dramatisée à outrance, au groupe compact des apôtres dont les barbes et les chevelures s'agitent en bouillonnantes virgules. À l'arrière-plan, un rayon phosphorescent inonde les remparts de Jérusalem tandis que la façade de la cathédrale Saint-Pierre reste dans l'ombre. On comprend qu'avec ses traits archaïques et son esthétique arbitraire, empreinte des artifices de la gravure flamande de la fin du XVI siècle, l'œuvre ait pu rapidement déplaire à la sensibilité de l'époque classique. En dépit de son importance quant à la dédicace du sanctuaire et à la place de l'ordre dans les sacrements de l'Église, c'est sans doute dès 1733, date du déplacement de l'autel majeur, qu'on remisa à la sacristie ce remarquable témoin d'un art désormais passé de mode.

8 En 1620, l'artiste se vit chargé, pour quatre mille livres tournois, de l'ornement de la voûte du vieux chœur roman pour laquelle il travailla à une Transfiguration et à d'autres thèmes. Comme la Pentecôte de la coupole, peinte par Louis Alexandre, Vincent Béquin et Charles Mousset en 1624, la Transfiguration d'Errard fut badigeonnée pendant la Révolution et redécouverte en 1834. Très abîmées mais encore lisibles, ces peintures murales, capitales pour la compréhension des grands décors religieux en province, disparurent à la fin du $\mathrm{XIX}^{\mathrm{e}}$ siècle $^{9}$ lors de la réfection du chœur de la cathédrale. Le décorateur Louis Viau en avait pris un relevé ${ }^{10}$ qui demeure pour l'instant introuvable.

Nommé peintre ordinaire du roi, Errard se rendit en 1615 et 1621 à Paris où il comptait certainement jouir de sa position et briguer quelque importante commande auprès de Marie de Médicis. Mais de plus grands artistes échouèrent là où réussirent la diplomatie et le génie de Rubens, et le peintre dut renoncer à ses ambitions. À Nantes, il exécuta en 1622 pour la chambre des comptes un « pourtraict au naturel [de] la personne de Sa $\mathrm{M}^{\text {té }}$ à cheval en un grand Tableau tel qu'il a esté posé pour se voir à presant au bas de vostre grand bureau... $»^{11}$. Charles Errard devait pourtant encore voyager en Italie où il accompagna son fils, futur directeur de l'Académie, périple durant lequel il rencontra Claude Gellée. Son autoportrait de $1628^{12}$ nous livre, dans un cadre abondamment orné, l'image d'un architecte couronné de lauriers et maniant mélancoliquement son compas ; c'est pourtant essentiellement à la peinture que le nom des Errard doit d'avoir survécu ${ }^{13}$, et avec cette Remise des clefs, la cathédrale Saint-Pierre recèle l'une des pages les plus parlantes du dernier maniérisme français. 
Figure 2

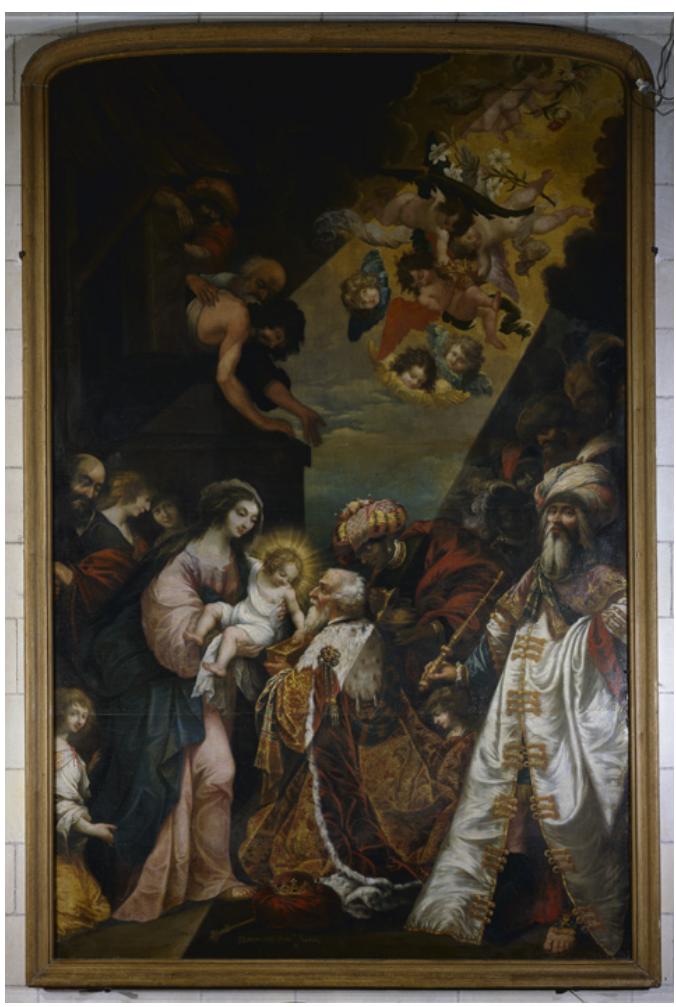

Mathurin Bonnecamp. L'Adoration des Mages. Nantes, cathédrale Saint-Pierre.

Phot. D. Pillet. (c) Région Pays de la Loire - Inventaire général, 8544 224VA.

10 La grande Adoration des mages autrefois dans la chapelle Saint-Jean-Baptiste, aujourd'hui dans la chapelle de la sacristie, a longtemps passé pour une «copie flamande du XVIII ${ }^{\mathrm{e}}$ siècle »; il s'agit en fait du seul tableau repéré, à notre connaissance, d'un artiste qu'identifie sa signature : «Bonnecamp pinci ${ }^{t} \operatorname{In}[v]$ eniat ${ }^{t}$ ( (fig. 2). On doit peut-être le rapprocher de Mathurin I Bonicamp (ou Bonican) signalé par Granges de Surgères ${ }^{14}$ comme portraitiste du $42^{\mathrm{e}}$ maire de Nantes (1634-1636), André Dubot sieur de la Grande Haye. Si l'on accepte cette hypothèse, l'origine nantaise de cette belle toile ne fait presqu'aucun doute et sa probable présence à la cathédrale avant la Révolution peut en expliquer l'ample format. La chatoyante Adoration des mages trahit maints emprunts aux grands peintres du début du XVII siècle : il y a du Claude Vignon et du Georges Lallemant ${ }^{15}$ dans ces rois pittoresquement enturbannés et parés d'atours rutilants, dans ces grappes d'angelots joufflus et dans le remous baroque de toute la composition. Pour des raisons de style, une datation du tableau autour des années 1630-1635 paraît plausible, d'autant qu'on peut lui comparer une Épiphanie signée « N. Lagouz Pinxit $1636^{16}$ ", vraisemblablement peinte à Angers, qui semble s'en inspirer largement. Le même rai de lumière barre la scène en la dynamisant « dans un genre un peu théâtral ${ }^{17}$ " et une disposition très similaire reprend l'attitude des personnages (dont certains sont des portraits), poncif il est vrai, inspiré des exemples italiens ou flamands (Rubens, Jordaens...). Mais le pinceau de Bonnecamp dénote une faconde qu'ignore Lagouz, plus retenu et malhabile, et confère au tableau de Nantes une aisance décorative qui nous fait regretter sa rareté.

11 On ignore l'origine du Saint Jean-Baptiste de François Lemoyne (1688-1737), signé et daté de 1715, mais son format, sa situation dans la chapelle des fonts baptismaux et le 
magnifique retable qui l'enchâsse depuis le début du XIx ${ }^{e}$ siècle incitent à le considérer comme l'un des tableaux ayant survécu aux destructions de la Révolution ${ }^{18}$. Très sousestimé autrefois, il importe aujourd'hui de mieux regarder cet unique témoignage à la cathédrale d'une peinture qui, pour n'être plus tout à fait classique, n'appartient pas encore au courant rocaille. Aussi bien l'influence de Raphaël, sensible dans la composition, s'accomode-t-elle ici de notations stylistiques indépendantes des grands modèles de l'iconographie religieuse. Le traitement très superficiel de l'image, scintillante de couleurs et de lumière, l'évocation d'une nature opulente et gracieuse et la beauté presque païenne du saint reflètent bien la maturité de l'artiste, l'harmonie de ses conceptions et la suprématie de son talent de décorateur.

Dans la première chapelle du bas-côté sud, le fragment d'une peinture murale représentant saint René, vraisemblablement du début du xvIII siècle, peut dater l'époque du bûchage des retables en pierre du $\mathrm{XvI}^{\mathrm{e}}$ siècle qu'il recouvre partiellement. Surtout, ses qualités naïves et fort provinciales démontrent a contrario l'ambition d'éventuelles commandes passées à La Fosse ou Lemoyne, futur peintre du roi. Mais au xvIII siècle et pour le peu qu'on en sache, le chapitre de la cathédrale semble aussi avoir fait appel à des artistes locaux, comme le suggère le tableau détruit de LouisFrançois Loysel (1740-1780) figurant le portail central de Saint-Pierre. Selon une coutume très répandue, la cathédrale conservait sans doute des copies d'après les maitres au nombre desquelles un "Lavement des pieds d'après Coypel ${ }^{19}$ [...] d'allure quelque peu fastueuse et solennelle ${ }^{20} »$. Quant à la médiocre Piéta de la chapelle du Sacré-Cœur et du Saint-Sacrement, nous sommes enclins à n'y voir qu'une maladroite adaptation pastichant des gravures du $\mathrm{xVII}^{\mathrm{e}}$ siècle (d'après Champaigne, Le Brun, Mignard...), datable des années de la Restauration.

Le bilan d'une prospection de la peinture ancienne à la cathédrale n'est pas si mince qui recense quatre tableaux, dont deux œuvres uniques, un fragment de décor mural et plusieurs mentions dans les textes. S'il contribue trop modestement à une meilleure connaissance de la peinture à Nantes avant la Révolution, ce bref inventaire n'exclut pas l'espoir de compléments ultérieurs, indispensables à une bonne compréhension des commandes du XIX siècle.

\section{Les peintures du $\mathrm{XIX}^{\mathrm{e}}$ siècle}

L'étude des tableaux d'une cathédrale implique un certain pragmatisme, comme l'analyse de toute collection constituée au fil du temps, au gré des commanditaires, des caprices de la mode, des politiques d'acquisition ou des impératifs d'une iconographie spécifique. Pourtant, Saint-Pierre de Nantes recèle un ensemble beaucoup moins disparate qu'il n'y paraît de prime abord. Essentiellement décorée dans la première moitié du XIX ${ }^{e}$ siècle (44 tableaux sur 51), la cathédrale offre au visiteur un remarquable échantillonnage des courants picturaux de l'époque. Certes, on n'y trouve pas les noms des plus grands : ni Géricault, ni Delacroix, ni Ingres n'ornent aujourd'hui l'intérieur de Saint-Pierre. On peut néanmoins y admirer quelques exemples insignes d'une production qui ne déparerait pas les salles du musée des Beaux-Arts de la ville. Mais dans l'édifice pour lequel ils ont été demandés ou conçus, les tableaux de Flandrin, Van den Berghe, Mauzaisse ou Delaunay sont chez eux, et disent mieux le formidable renouveau de la peinture religieuse de la période, désormais bien éclairé par l'ouvrage de M. Bruno Foucart ${ }^{21}$. 
15 À Nantes, nécessité fit loi où béaient les chapelles dépouillées par la Révolution et peutêtre aussi par l'incurie et l'inconscience constatables en maints autres sanctuaires à la fin du XVIII ${ }^{e}$ siècle. La remise en état de la cathédrale alla bon train sous l'impulsion des prélats, Mgr Micolon de Guérines notamment, avec quelques décennies fastes, celles des années 1820 à 1850, durant lesquelles l'édifice s'enrichit de la majorité des peintures qui s'y trouvent encore. Du seul Salon de 1839, le nouvel évêque Mgr de Hercé parvient à obtenir trois œuvres (Donné [n ${ }^{\circ}$ 593], Doussault [ $\left.{ }^{\circ} 595\right]$, Vauchelet [ ${ }^{\circ}$ 2042]). D'autre part, l'élaboration hésitante des décors des chapelles Saint-Clair et du Sacré-Cœur témoigne de l'attention dont les autorités ecclésiastiques firent preuve dans leur choix du cadre visuel des dévotions et des cultes. L'exigence d'une cohérence des thèmes confère au programme une belle unité d'esprit mais trahit aussi les préoccupations d'un clergé qui oscille entre ré-évangélisation et mémorialisme romantique: les martyrs de l'Église traditionnelle soutiennent le discours avec trop d'insistance pour qu'on ne perçoive là une célébration de plus récents sacrifices. Enfin, la cathédrale est un lieu d'exposition permanente où les peintres peuvent démontrer leur talent à travers de grandes et ambitieuses compositions historiques. Du « grand style » de Flandrin, raillé par Delacroix ${ }^{22}$, aux essais moins heureux de quelques artistes locaux, c'est une inappréciable part du débat pictural de deux générations, leurs réussites et leurs limites, qu'il est permis d'embrasser d'un seul regard en parcourant Saint-Pierre; esquisse elliptique d'un musée idéal auquel ne manque qu'une grande réalisation murale ${ }^{23}$ pour présenter toute la trajectoire de la peinture religieuse au XIX ${ }^{e}$ siècle.

\section{La chapelle du Sacré-Cœur et du Saint-Sacrement}

16 Les exemples de la chapelle du Sacré-Cœur et de celle de Saint-Clair illustrent parfaitement ce que furent les difficultés pour remeubler et redécorer le sanctuaire et l'extrême versatilité des choix fluctuant selon des commandes interchangeables et des atermoiements sans fin. Il semble qu'à Paris, l'administration des cultes ait eu beaucoup de peine à répondre aux sollicitations : «Je reçois chaque jour de la part de MM. les évêques, préfets et membres de la Chambre des Députés, des demandes de tableaux représentant des sujets de dévotion, pour décorer les cathédrales [...] Le peu d'encouragements que cette partie de l'art a reçu en France depuis plus de trente ans, fait que les collections de Votre Majesté sont entièrement dépourvues des moyens de venir sous ce rapport, au secours du culte" écrit en 1825 le vicomte de La Rochefoucauld ${ }^{24}$.

Pour Nantes ${ }^{25}$, le comte Auguste de Forbin, directeur des Musées, avait demandé une peinture à Théodore Géricault (1791-1824) en décembre 1819, et dès le 12 janvier de l'année suivante, une ordonnance royale - réservant soixante mille francs pour les églises du royaume - allouait au peintre une somme de deux mille quatre cents francs pour un tableau destiné à la cathédrale, et non au couvent des Dames du Sacré-Cœur comme il a souvent été dit $^{26}$. En avril $1820^{27}$, l'architecte départemental précisait l'emplacement (au nord) et le sujet de l'œuvre décidé par l'évêque, une Dévotion du Sacré-Cœur de Jésus et de Marie. Ce thème déplut-il à Géricault? Se sentait-il déjà trop malade pour l'entreprise, ou ses séjours en Angleterre l'en empêchaient-ils ? Quoi qu'il en fût, Géricault transmit la commande à son jeune ami Eugène Delacroix (1798-1863) en juin 1820 et convint avec lui d'en garder la responsabilité (c'est-à-dire de signer la toile) et d'en partager le revenu. Delacroix se mit rapidement à l'ouvrage ; une lettre à 
sa sœur évoque les esquisses ${ }^{28}$ qu'il dut soumettre à l'approbation de l'évêque. La correspondance avec Pierret et Soulier durant l'hiver 1820-1821 atteste les nombreuses difficultés rencontrées par l'artiste, continuellement insatisfait de l'élaboration de sa composition. Enfin terminée vers la fin de 1821, la toile ne fut livrée et payée qu'en mai et août 1822, et la caisse parvint à Nantes le 2 novembre.

Le tableau avait peut-être déçu Géricault - mais le fait n'est pas très sûr -, il n'obtint pas en tout cas l'agrément de ses destinataires qui jugèrent que « loin d'être religieux et d'édifier les fidèles, il ne pouvait servir qu'à les scandaliser et à leur inspirer des idées et des sentiments contraires à la religion et à la pudeur ${ }^{29}$ ». La Vierge du Sacré-Cœur resta néanmoins à Nantes jusqu'en janvier 1824, date à laquelle le préfet écrivit au ministre : «Je partage l'opinion de Mrs. les vicaires et vous invite à prendre les mesures nécessaires pour que le tableau dont il s'agit soit renvoyé à Paris $»^{30}$. Mgr Micolon de Guérines demanda en 1826 au ministre de l'Intérieur un Saint Clair en remplacement du Sacré-Cœur de "feu M. Géricault ", mort en 1824. Cette substitution suggère un échange, une sorte de marché, bien que les deux œuvres n'aient pas été prévues pour une même chapelle. Le tableau de Delacroix ne réapparut qu'en 1827, lors de son installation à la cathédrale d'Ajaccio, toujours sous sa fausse identité, et la supercherie ne fut révélée qu'au milieu du siècle. Le refus du Géricault-Delacroix pose de multiples problèmes que n'élucide pas sa seule "indécence » aux yeux de ses censeurs nantais et il est possible que son format n'ait pas convenu au local : Delacroix évoque parfois cette question des dimensions dans sa correspondance. La seule évidence qui demeure est celle d'une occasion manquée par Nantes, dont la cathédrale ne peut aujourd'hui s'enorgueillir, d'un des premiers grands tableaux du plus important des romantiques français.

Et comme on ne parvient pas à trouver un Sacré-Cœur convenable, on se préoccupe, dès 1826, d'avoir au moins un Saint-Sacrement. Le sujet sera abordé à travers le thème de la communion donnée par saint Charles Borromée aux pestiférés de Milan, peut-être parce que la cathédrale possédait auparavant un retable dédié à saint Charles et qu'on voit là le moyen d'honorer un ancien vocable tout en illustrant le nouveau... C'est à Jean-Baptiste-François Désoria (1758-1832) qu'échoit la commande, prestement honorée puisque son tableau porte la date de 1827 (fig. 3). Une lettre du 27 décembre 1827 du ministre au préfet dessine l'étroite imbrication des commandes à cette époque: "Le tableau représentant St-Charles Borromée que j'ai accordé à Monseigneur l'évêque pour sa cathédrale vous est adressé et vous parviendra sous peu de jours par routage. Ce tableau remplacera à la cathédrale celui de la Vierge du SacréCœur précédemment accordé et dont j'ai disposé en faveur d'une autre cathédrale ${ }^{31}$. Ainsi qu'il est d'usage en pareille circonstance, les frais d'encaissement et de transport devront être imputés sur les fonds à votre disposition. Les frais de la matière que pourra occasionner dans quelques mois l'envoi du tableau de St-Clair aussi destiné à la cathédrale de Nantes seront également imputés sur les fonds précités ${ }^{32}$. 
Figure 3

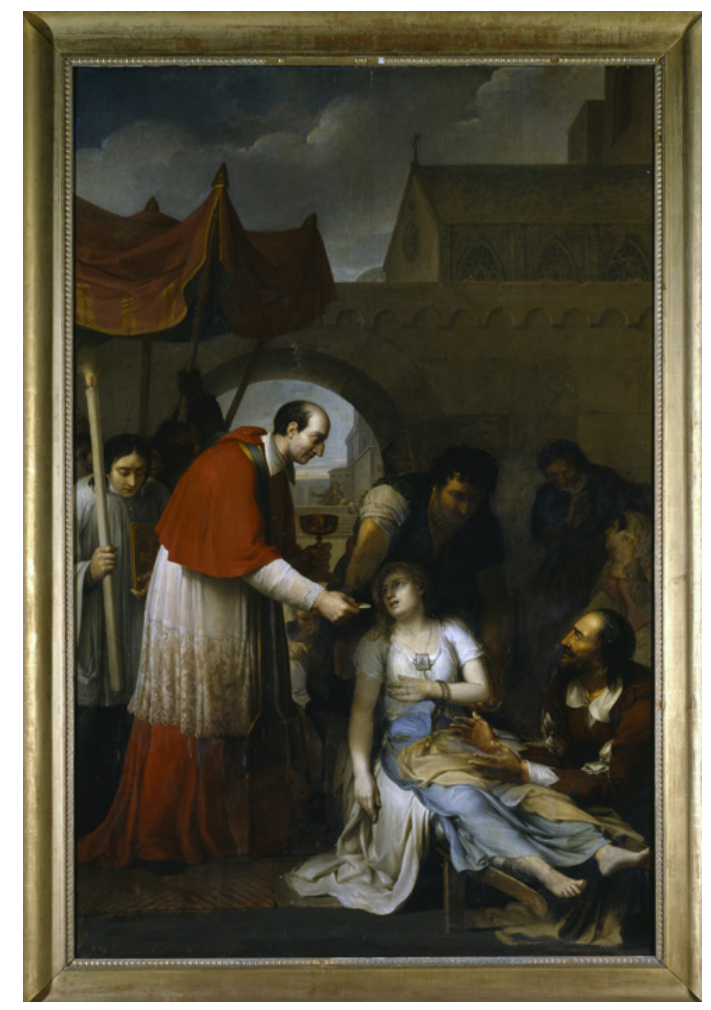

Jean-Baptiste-François Désoria. Saint Charles Borromée communiant les pestiférés de Milan. Nantes, cathédrale Saint-Pierre.

Phot. D. Pillet. @ Région Pays de la Loire - Inventaire général, 8544 213VA.

Alors à la fin de sa carrière, Désoria offrait certes les garanties d'un sérieux et d'un savoir-faire académique éprouvé. S'il s'était mieux distingué dans l'art du portrait - on admire encore celui d'Élisabeth Dunoyer qui inspira David ${ }^{33}$ - cet élève de Jean-Bernard Restout n'était pas parvenu à se faire véritablement un nom dans la peinture d'histoire. Désoria, artiste quasi itinérant, professa à Évreux, Rouen, Metz, Cambrai ; peintre éclectique ou pédagogue sans racines, on ne peut nier en tout cas ses évidentes qualités d'assimilation et de synthèse. L'inspiration de la toile de la cathédrale de Nantes puise ainsi aux innombrables sources visuelles léguées par la tradition du thème aux XVII et XviII ${ }^{e}$ siècles : le Saint Charles Borromée de Pierre Mignard (Le Havre, musée des Beaux$A r t s)^{34}$ par exemple, qui développe une mise en scène des protagonistes dans un décor architectural, une immédiateté de l'image et un sens du drame classique fort comparables dans leurs principes au tableau nantais. Pourtant, l'intelligence maîtrisée des emprunts (Poussin, Carrache, Cortone, Maratta...), souvenirs d'un séjour à Rome à la fin des années 1780, masque mal l'indigence fade de l'exécution et son habileté inopérante.

Restait en suspens la question du Sacré-Couur. Durville ${ }^{35}$ affirme que « Bézart » en aurait exécuté un pour la cathédrale. En ce qui concerne l'artiste, il ne peut s'agir que du peintre Jean-Louis Bézard (1799-1881), mais l'œuvre, elle, semble avoir disparu ou même n'avoir jamais existé. À moins qu'il faille attibuer à Bézard les Deux anges avec le Saint-Sacrement, décor mural de la chapelle de la bienheureuse Françoise d'Amboise, probable témoignage d'un projet abandonné pour une chapelle du Sacré-Cœur et du Saint-Sacrement, avant les grands travaux de la seconde moitié du siècle et un 
changement de dédicace. La bibliographie et les archives restent pour l'instant muettes sur ces deux beaux anges qui évoquent ceux que Bézard brossa en 1844 au dessus du Christ mort sur les genoux de la Vierge à Sainte-Élisabeth à Paris. L'artiste aima représenter ces symboliques acteurs ailés, sublimant des « sujets peu théologiques mais appartenant à la religion du cœur ${ }^{36}$ ». Finalement, le décor de la chapelle du SacréCœur et du Saint-Sacrement sera de nouveau remanié, une quarantaine d'années après sa création, et son vocable illustré d'une copie de Sotta, pour présenter le Sacré Cœur, et de la Communion des apôtres de Delaunay, remplaçant désormais le Saint Charles de Désoria.

\section{La chapelle Saint-Clair}

Avant les déprédations de la Révolution, la première chapelle du bas-côté sud, près de l'autel, était dédiée au Sauveur et aux saints Lazare, Marthe et Marie. Sous la Restauration ${ }^{37}$, on abandonna l'idée d'un thème qui eût rappelé ceux de l'ancien décor et dès 1820 , le duc Decazes promit un tableau. Une lettre de l'évêque au ministre (par l'intermédiaire du préfet), en novembre 1821, proposait une iconographie consacrée à saint Clair, premier évêque de Nantes (fig. 4). La même année, le ministère de l'Intérieur choisit Jean-Baptiste Mauzaisse (1784-1844) pour cette commande rétribuée d'une somme de six mille francs ${ }^{38}$. Le sujet de la peinture commandée, le saint prélat rendant la vue à un aveugle, mettait en avant une volonté nettement prosélytique au détriment d'une simple restauration du cadre cultuel de l'Ancien Régime. L'histoire de Nantes pendant les troubles de la Révolution justifiait assez pour le clergé une véritable reprise de l'évangélisation dans un esprit contre-réformateur marqué. En mai 1826, une lettre de l'architecte départemental Ogée évoque le plan de la chapelle et son élévation " où se voit le tableau de saint Clair, avec (ses) dimensions" tandis qu'en juin de la même année, Mgr Micolon de Guérines réitère sa demande au ministre en arguant de la perte du Sacré-Cœur de "Géricault». Enfin, en juillet 1826, une lettre de Bodinier, vicaire général, écrite pour l'évêque au ministre de l'Intérieur, nous apprend que l'œuvre de Mauzaisse était presque achevée mais qu'on aurait désormais préféré un Saint Charles Borromée distribuant la sainte Communion aux pestiférés de sa ville archiépiscopale. En fait, le décor de la chapelle du Sacré-Cœur et du Saint-Sacrement était devenu prioritaire. 
Figure 4

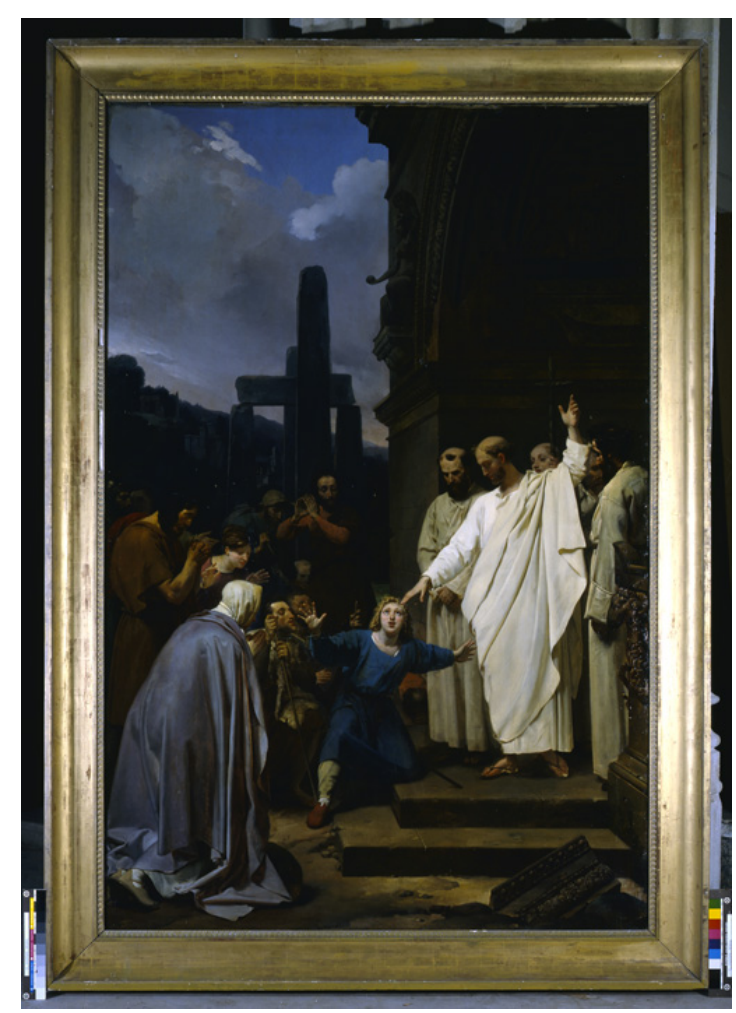

Jean-Baptiste Mauzaisse. Saint Clair rendant la vue à un aveugle. Nantes, cathédrale Saint-Pierre.

Phot. D. Pillet. @ Région Pays de la Loire - Inventaire général, 8544 222VA.

Le paiement du Saint Charles Borromée de Désoria fut-il la cause de l'éviction du Saint Clair de Mauzaisse? En tout état de cause, celui-ci n'acheva son œuvre qu'en 1828 sans qu'on puisse saisir précisément les raisons de ce retard. Les sources documentaires restent fort confuses, voire contradictoires, en ce qui concerne son accrochage effectif dans la cathédrale et il est permis de douter qu'il y fût jamais visible. En mars 1837, une lettre $\mathrm{du}$ conseil de fabrique et du chapitre à l'évêque déplorait en effet le dépouillement de la chapelle et l'on remplaça directement un vieux tableau anonyme représentant saint Clair par celui de Flandrin. Dans ces années 1827-1828, Mauzaisse se trouvait au fait de sa notoriété, travaillant au Louvre au plafond de la salle Louis XVIII et à celui de la salle dite du trésor de Boscoreale pour lequel, en 1822, il avait déjà brossé une puissante Allégorie du Temps montrant les ruines qu'il amène et les chefs-d'œuvre qu'il laisse ensuite découvrir ${ }^{39}$. L'idée du fragment d'entablement antique au premier plan du tableau de Nantes, d'une signification symbolique évidente, provient directement de l'allégorie du Louvre. D'importantes commandes publiques parisiennes accaparaient donc l'activité de l'artiste lorsqu'il peignait le Saint Clair de la cathédrale, explication possible de son retard mais signe aussi de l'ambition du clergé nantais et de sa volonté d'obtenir une œuvre d'un des peintres les plus en vue. De fait, la composition surpasse en qualité ses autres tableaux religieux, d'une veine moins inventive et sensible, qu'il s'agisse du gigantesque Martyre de saint Étienne (Salon de 1824, autrefois à la cathédrale de Bourges, transféré au Louvre) ou du Saint Vincent Ferrier de la cathédrale de Vannes (Salon de 1831). La figure de saint Clair, comme celle d'un moderne Le Sueur, tire sa force monumentale du rythme ample des drapés à l'antique et d'une rhétorique mesurée des gestes. Idéalisme, mais aussi réalisme de la facture extrêmement soignée, 
exalté par les contrastes de la vive lumière dont l'artiste baigne la scène. Sur fond de mégalithes bretons et de temples païens, les personnages esquissent une chorégraphie des plus déliées, animée à gauche par l'étrange ballet des mains, pondérée à droite par le robuste statisme du saint évangélisateur et de ses compagnons. Deux grandes diagonales invisibles relient ces deux pôles et convergent en chiasme sur le visage illuminé de l'adolescent miraculé. La forte éloquence de Mauzaisse dépasse, et de loin, cette facile parabole de la cécité vaincue par l'Évangile.

En 1833, un pieux bienfaiteur ayant fait un don à l'abbé d'Audrain, celui-ci souhaita consacrer cette somme au décor de la chapelle Saint-Clair. À la fin de l'année suivante, l'architecte Moll, qui se verrait offrir une esquisse du tableau (conservée au musée des Beaux-Arts d'Angers) et le peintre Bodinier, apparenté au vicaire général, parvinrent à orienter la commande sur leur jeune ami Hippolyte Flandrin (1809-1864). En septembre 1835, celui-ci écrivait à son frère Auguste :

J'ai à faire un tableau pour la magnifique Cathédrale de Nantes [...] dont saint Clair était l'évêque au troisième siècle, et la toile a neuf pieds de haut. Ce sont MM. Bodinier qui ont arrangé ça. Ils m'ont proposé ce tableau, et j'ai accepté avec grand plaisir, pour faire au moins quelque chose qui ait un emploi. Quant au prix, il n'en faut pas parler. Je fais quinze figures grandes comme nature pour mille francs, à peu près le montant des frais $[. . .]^{40}$. Rome et c'est en qualité d'envoi de troisième année qu'il fut montré à Paris, à l'École des beaux-arts, en août 1836 (fig. 5).

\section{Figure 5}

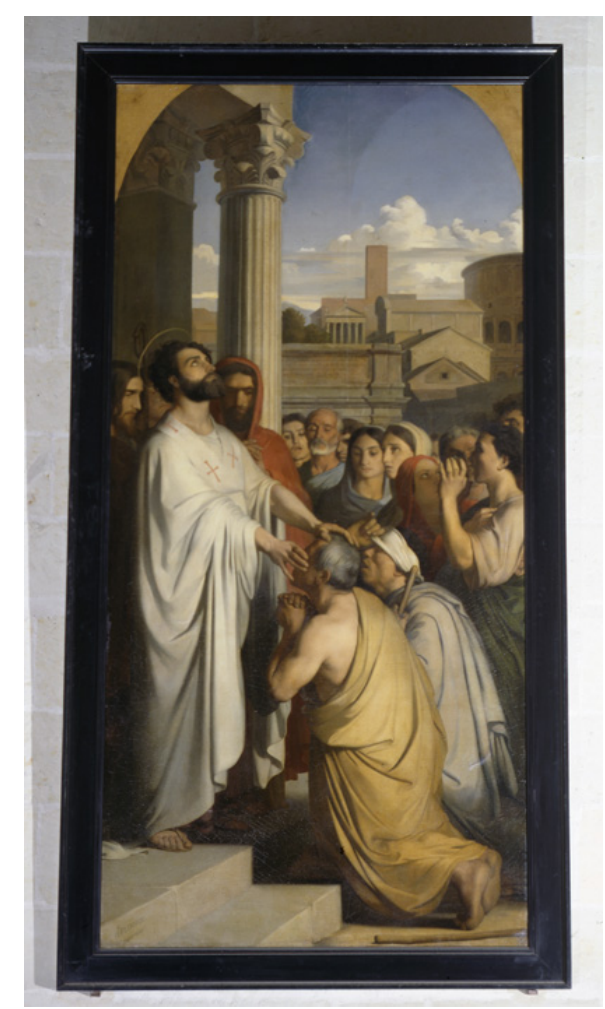

Hippolyte Flandrin. Saint Clair rendant la vue à un aveugle. Nantes, cathédrale Saint-Pierre. Phot. D. Pillet. ( Région Pays de la Loire - Inventaire général, 19914401280 XA. 
mière œuvre religieuse importante de Flandrin, régulièrement exposée à Paris au cours du siècle $(1837,1855 . .$.$) , le Saint Clair valut à son auteur plusieurs médailles et$ suscita une abondante littérature où s'entremêlaient louanges et reproches. Ceux de Théophile Gautier furent les plus humoristiques qui suggéraient «qu'un tableau de saint Clair ne devait pas être si sombre $»^{41}$. La confusion des plans, la froideur du coloris, la trop fidèle adaptation de modèles reconnus, ceux de Giotto, de Raphaël ou d'Ingres, indisposèrent certains commentateurs mais globalement, la critique ultérieure sut reconnaître dans le tableau de Nantes les prémices des réalisations les plus magistrales du maître.

27 À l'instar des décors muraux où Flandrin excellera, la composition se dépouille ici de toute afféterie et réduit la narration à sa plus directe expression. Aux volumes classiques et épurés de l'architecture antique ${ }^{42}$ répondent ceux, plus denses encore, des toges et des visages des personnages. On y reconnaît le portrait du musicien Ambroise Thomas en disciple du saint ${ }^{43}$, hommage du peintre à son ami et seule concession de circonstance à l'essence désincarnée de la scène. À Rome, Ingres en perçut toute la force :

Il est entré, s'est placé en face du tableau. Assis depuis un moment, il ne disait rien, j'étais embarrassé, Paul aussi. Enfin, il se lève, me regarde, les yeux pleins de larmes, et, en m'embrassant avec effusion, le sentiment que vous lui connaissez, il me dit: «non, mon ami, la peinture n'est pas perdue. Je n'aurai donc pas été inutile... $»^{44}$.

Le décor dans lequel le tableau s'insérait comportait en outre un vitrail, également sur le thème de saint Clair (1837), des boiseries néo-gothiques dessinées par Liberge, et neuf peintures de Jules Laure (1806-1861) : le Christ, la Vierge ${ }^{45}$, saint Jean et les saints patrons des paroisses de la circonscription de Nantes sous l'Ancien Régime. Cinq des neuf tableaux de Laure viennent d'être sauvés de la destruction à laquelle les vouait lentement leur abandon dans la crypte de la cathédrale. La monumentalité des figures devait particulièrement bien répondre au classicisme de Flandrin ; quant aux qualités de la réalisation, que la restauration actuelle révèlera bientôt, elles sont comparables à celles des médaillons (des saints et des anges) que l'artiste laissa à Saint-Vincent-dePaul à Paris. Le peintre maintint sans doute quelques rapports avec Nantes où, en $1854^{46}$, il exposa plusieurs portraits, domaine dans lequel il avait acquis une relative notoriété.

ne Sainte Philomène, qui aurait été commandée à Jalabert, est également décrite avec précision dans la notice de 1845 sur la chapelle Saint-Clair, mais cette mention procède d'une erreur, voulue ou non, dans l'interprétation de l'iconographie ${ }^{47}$. L'œuvre récemment retrouvée porte en fait la date de 1858 et la signature d'un certain L.E. de La Boëssière. Le " triptyque » constitue en tout cas un bon exemple du goût néo-gothique respectant le style architectural de Saint-Pierre.

Inaugurée en 1845, la chapelle servit désormais principalement aux cérémonies paroissiales ; elle témoignait alors, dans l'intégrité et l'homogénéité de son décor, de la primauté renouvelée que le clergé nantais entendait attribuer à l'image et au symbole.

\section{Deux « primitifs »}

31 Les années 1838 et 1839 s'avèrent cruciales pour appréhender la nature avant-gardiste des acquisitions et des commandes de tableaux pour Saint-Pierre. Au XIX ${ }^{\mathrm{e}}$ siècle, 
Nantes, dont le musée des Beaux-Arts accueillait, de leur vivant, des œuvres majeures de grands artistes contemporains (Ingres en 1853, Courbet en 1861), Nantes où le renouveau spirituel cristallisait l'émergence du néo-gothique (l'église Saint-Nicolas, de Lassus, date des années 1840), Nantes enfin ne pouvait manquer pour le décor de sa cathédrale certains choix risqués mais fort révélateurs d'une option tout à la fois novatrice dans sa forme et conservatrice sur le fond. L'installation du Saint Clair de Flandrin vers 1838 , premier signe de ce regain de ferveur militante, annonce l'ouverture à des tenants du goût primitif et un retour aux valeurs de l'art d'avant la Renaissance, quand Raphaël ne supplantait pas encore Pérugin.

Figure 6

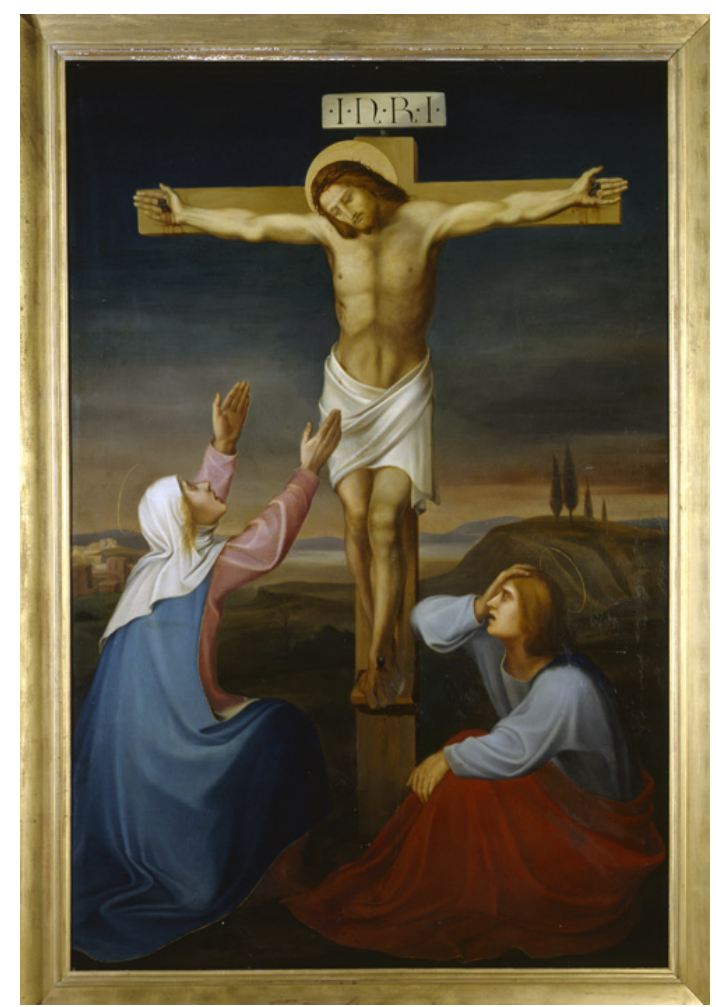

Édouard Hauser. La Crucifixion avec la Vierge et saint Jean. Nantes, cathédrale Saint-Pierre. Phot. D. Pillet. (c) Région Pays de la Loire - Inventaire général, 8544 217VA.

L'étrange Crucifixion d'Édouard Hauser (1807-1864) (fig. 6) participe de ce courant et, pour déroutante et presque médiocre qu'elle puisse paraître aujourd'hui, n'en précise pas moins un ressourcement marqué de l'imagerie religieuse. De ce peintre suisse installé à Paris en 1837 sous la protection de Montalembert, la plus utile contribution au débat artistique français fut sans conteste d'y introduire un peu de l'influence des nazaréens allemands ${ }^{48}$. Lié à Overbeck et Cornelius, au père Danzas (membre fondateur de la Société de Saint-Jean), Hauser relie les sectateurs germains de l'Angelico et ses admirateurs français. Telle Déploration sur le Christ mort de Savinien Petit (1839) ${ }^{49}$, autre confrère de la Société de Saint-Jean, semble l'écho parisien de la Crucifixion de Nantes par la simplification des volumes et l'insistance dramatique des effets lumineux. Mais au lieu de l'inspiration italienne chère aux familiers du Casino Massimo ${ }^{50}$, Hauser puise au répertoire des primitifs colonais du XV $\mathrm{XV}^{\mathrm{e}}$ siècle, le Maître de la Vie de la Vierge ou le Maître du retable de Saint-Barthélémy ${ }^{51}$, empruntant à l'un sa ligne stylisée, à l'autre la 
véhémence de son expressionnisme. Seuls reliquats de douceur toscane ou ombrienne, les draperies ondulantes et le paysage hyper-simplifié de l'arrière-plan citent des modèles ultramontains de façon décalée, comme déplacée, sans emporter l'adhésion du spectateur. Encore manquons-nous d'éléments pour juger des sentiments du fidèle de 1838.

Figure 7

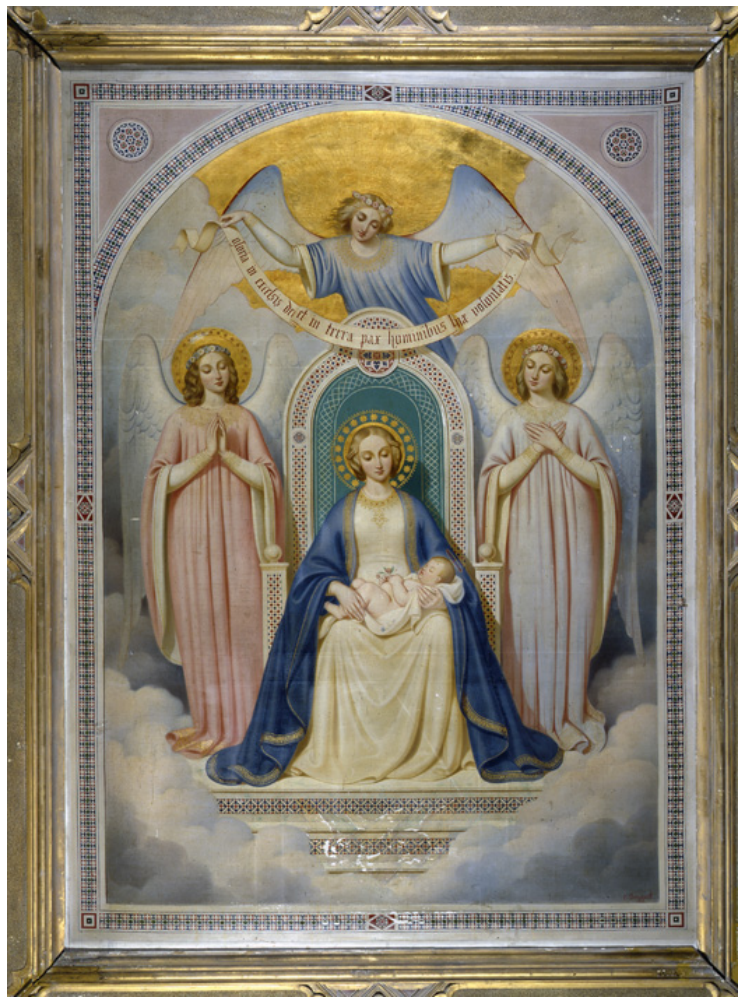

Charles Doussault. La Vierge aux anges. Nantes, cathédrale Saint-Pierre.

Phot. D. Pillet. @ Région Pays de la Loire - Inventaire général, 8544 229VA.

La Vierge aux anges de Charles Doussault (1806-ap. 1873) (fig. 7) porte la même date et fut choisie au Salon de 1839. De ce peintre fougerais partageant sa vie entre Paris et la Bretagne, on connaît surtout le goût pour des sujets pittoresques ou orientalistes (il en exposa à Nantes à plusieurs reprises, de 1842 à 1872) 52 $^{2}$ ainsi que quelques sculptures académiques telle sa Danseuse antiqu ${ }^{53} \mathrm{du}$ musée des Beaux-Arts. Hormis des débuts auprès des frères Devéria et avant ses voyages en Orient (1842) et en Italie (1854), la formation de l'artiste dut être influencée par l'archaïsme de Victor Orsel (1795-1850). En effet, comme les fresques de Notre-Dame-de-Lorette à Paris ou le Vœu du choléra de Notre-Dame de Fourvière à Lyon, le tableau de Doussault exploite une veine fortement teintée de quattrocento florentin: type physique adouci des anges et de la Vierge, frontalité du trône et des personnages, discrète utilisation du fond d'or... Il porte aussi en lui quelques traits d'un Moyen Âge plus national que lui confèrent la pastellisation de la gamme colorée et une bordure décorative géométrique à laquelle répond le cadre néo-gothique. Annonçant la fadeur " 1900 » d'une certaine production saintsulpicienne, cette œuvre un peu mièvre contribue pourtant, avec la Crucifixion de Hauser, à situer les acquisitions de la cathédrale de Nantes parmi les plus significatives de la période. 


\section{Les martyrs} ravages subis par le patrimoine de l'Église durant cette période (sans parler des crimes contre les hommes). Les guerres de Vendée avaient trop pris un tour de croisades pour qu'on ne ressentît pas, la Restauration venue, le besoin d'en prolonger les effets, signes d'une reconquête menée de l'intérieur sur les esprits et sur les cœurs. Le choix des sujets des tableaux de la cathédrale relève ainsi, nous l'avons évoqué pour la chapelle Saint-Clair, d'un parti ré-évangélisateur et d'une sensibilité presque post-tridentine. Tourné vers le passé, cet aspect intentionnel de l'iconographie catalyse, à Nantes, une apologétique triomphante du martyre. Le tiers des tableaux que nous inventorions exaltent par leurs thèmes une morale de la souffrance, du sacrifice corporel, de la mort consentie, exemplaire et rédemptrice. Stigmates des passions, au double sens du terme, et des malheurs de temps qu'on souhaitait révolus, les images à méditer dans la cathédrale décantent, sans qu'il soit nécessaire d'en pousser trop avant l'analyse, le traumatisme des persécutions. Moins expiatoire que politique, l'utilisation de thèmes sanglants ou funèbres tirés du passé catholique rappelle aux Nantais d'autres morts de plus fraîche mémoire et les re-présente à la réflexion des fidèles.

\section{Figure 8}

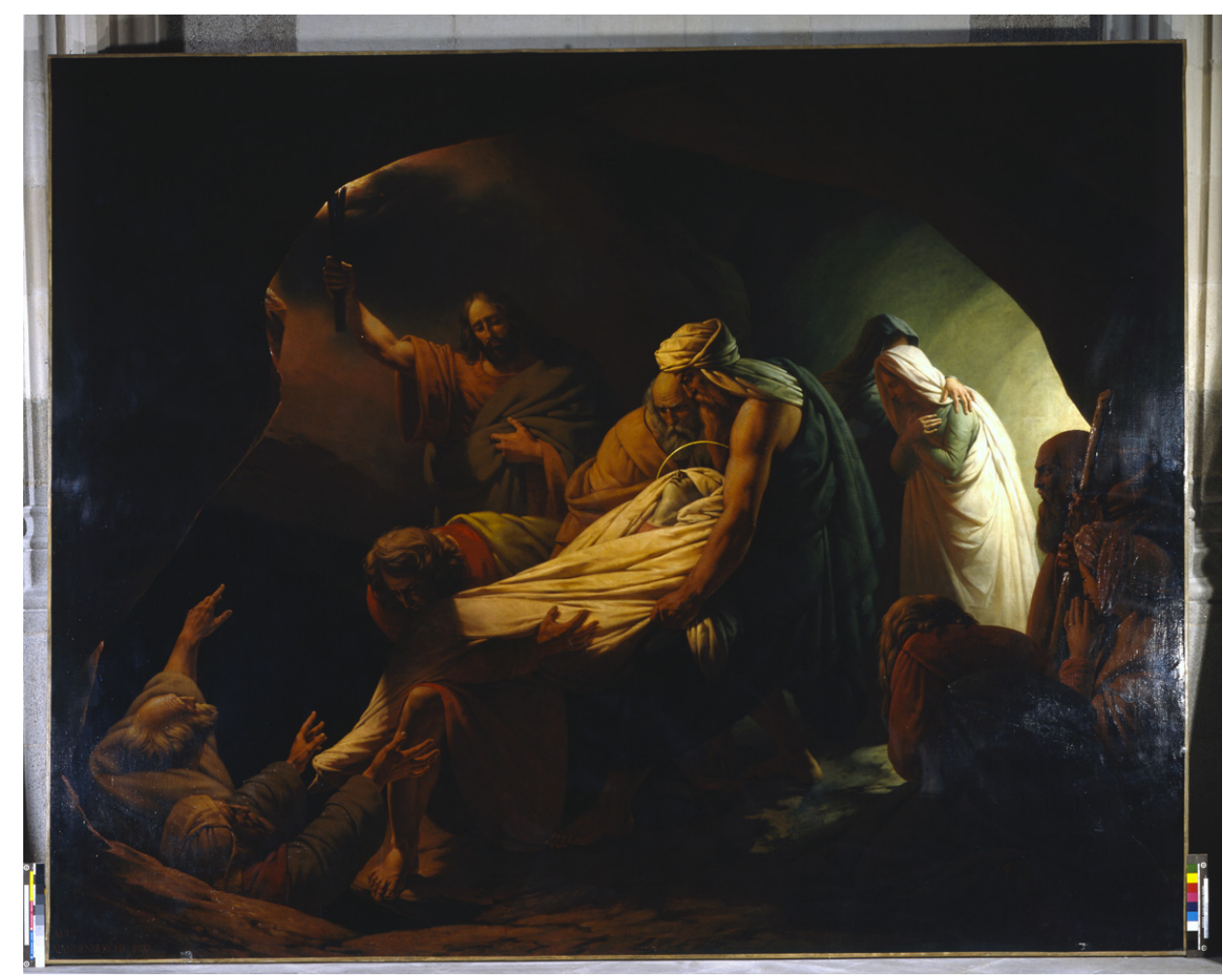

Charles-Auguste Van den Berghe. L'Ensevelissement de la Vierge. Nantes, cathédrale Saint-Pierre. Phot. D. Pillet. (c) Région Pays de la Loire - Inventaire général, 8544 223VA.

Le témoignage le plus remarquable de cette ambiance reste l'immense tableau de Charles-Auguste Van den Berghe (1798-1853), un Ensevelissement de la Vierge daté de 1835 et alloué à Nantes en septembre de l'année suivante (fig. 8). Fils d'un artiste belge 
d'une certaine réputation, professeur à la manufacture de tapisseries de Beauvais, Van den Berghe dut aux relations de son père d'accéder aux cours de quelques-uns de ses célèbres amis. Il suivit donc un parcours assez représentatif du cursus des aspirants peintres de la première moitié du XIXe siècle. Formé par Gros et Girodet à l'École des beaux-arts de Paris, émule de Guérin et Vernet lors de son séjour à Rome (1827-1829), bon violoniste comme Ingres, aucun des épisodes les plus convenus d'une vie d'artiste officiel de l'époque ne manque à sa très bourgeoise carrière, ni les succès au Salon, ni les engagements successifs dans la Garde nationale, ni même la Légion d'honneur. Il y a pourtant dans son œuvre de puissants moments d'inspiration, des failles où un fécond malaise s'insinue dans les compositions les plus conventionnelles, comme à l'encontre de la volonté du faiseur. Froide langueur des portraits ${ }^{54}$ ou classicisme édulcoré des tentatives pour le prix de Rome $e^{55}$, l'art du jeune Van den Berghe laisse néanmoins sourdre l'amertume des aspirations mal satisfaites de sa génération, une propension à la mort et aux nuits ${ }^{56}$ qui distille ses poisons dans l'impressionnante toile de la cathédrale.

Celle-ci fut présentée au Salon de 1836 et l'artiste désirait qu'elle décorât la cathédrale de Beauvais, « le lieu où j'ai été baptisé, où j'ai fait ma première communion. Ce sont de ces idées qui feraient rire bien des gens, mais ce ne sont pas moins mes idées » confiaitil à un intime en 1836. Mais "sur la demande spéciale d'une députation de Nantais, le roi acheta le tableau de Van den Berghe pour la cathédrale de Nantes, où on lui a assigné une place d'honneur $\aleph^{57}$. Au premier coup d'œil, L'Ensevelissement de la Vierge avoue la dette de l'artiste envers son maitre Girodet dont il pille franchement les Funérailles d'Atala ${ }^{58}$. Transposition du romanesque au sacré, soit, mais qui adopte respectueusement le schéma initial de sa source d'inspiration. Au centre de la scène, le rougeoiement de la torche sculpte le suaire d'une Vierge auréolée mais le visage voilé, et concentre le drame dans l'espace abyssal de la grotte qui lui sert d'écrin funèbre. Le procédé n'est pas neuf qui force la plasticité des formes en accentuant le contraste des ombres et des zones éclairées, pourtant, le tour de force d'éclectisme de Van den Berghe réside bien ici dans sa capacité à marier ténébrisme et néo-classicisme, intimité et grande peinture d'histoire. Une réussite qui frappa le commentateur de L'Univers religieux du 26 avril 1836, estimant l'artiste désormais «digne de voir son pinceau consacré à décorer nos églises $»^{59}$. 
Figure 9

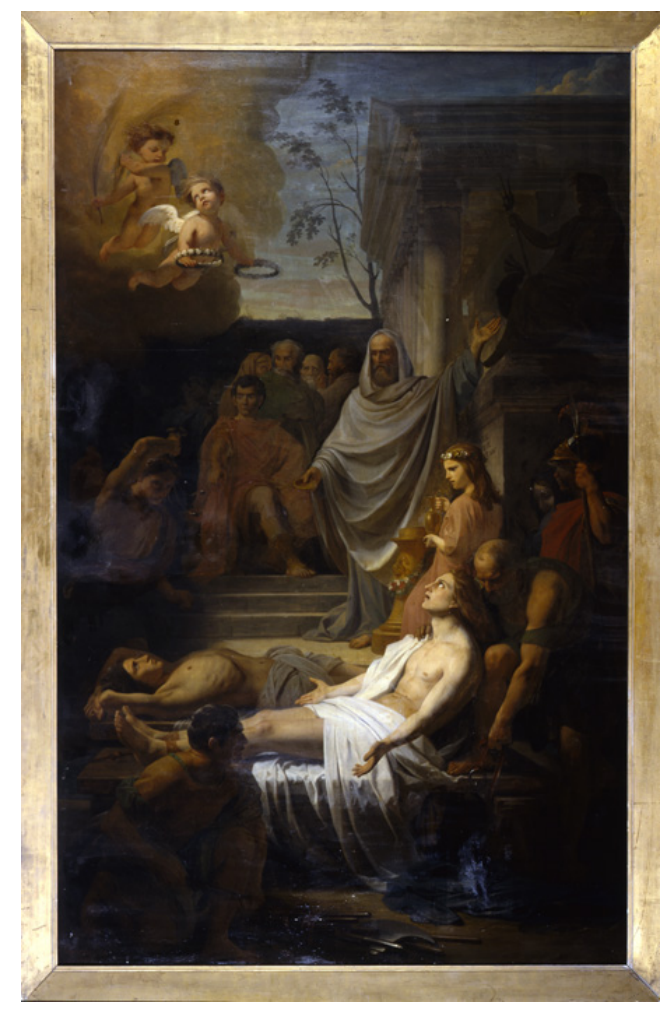

Théophile-Auguste Vauchelet. Le Martyre de saint Donatien et saint Rogatien. Nantes, cathédrale SaintPierre.

Phot. D. Pillet. (c) Région Pays de la Loire - Inventaire général, 8544 221VA.

Peu avant 1830, le chapitre avait souhaité qu'un Martyre de saint Donatien et saint Rogatien vînt rappeler à la mémoire des fidèles le sacrifice des deux Nantais ${ }^{60}$ et leurs reliques que conservait la cathédrale avant la Révolution. Théophile-Auguste Vauchelet (1802-1873) dut en recevoir la commande vers 1835, suite à une lettre de l'évêque au ministre réitérant sa demande d'un tableau sur ce thème ${ }^{61}$ (fig. 9), et présenta son œuvre au Salon de 1839. Prix de Rome dix ans auparavant, l'artiste avait essentiellement fondé l'essor de sa carrière sur de grands décors muraux qui lui valurent maintes récompenses mais que le temps n'a pas épargnés. Des ensembles parisiens de Saint-Germain-l'Auxerrois, de la chapelle du Luxembourg ${ }^{62}$ ou de l'Hôtel de ville, presque rien n'a survécu ${ }^{63}$, et cette déroute attache à l'ambitieuse peinture de la cathédrale un intérêt que ne justifient pas ses seules qualités. C'est une grande page d'un classicisme d'emprunt, encombrée de références à Poussin et Le Sueur, au dessin d'une froide correction et au coloris plombé. Vauchelet y dépeint sans passion le supplice accepté des deux Nantais dont une paire d'angelots vient couronner la jeune beauté sacrifiée. Aux bourreaux et aux juges sont réservées l'ombre et la laideur, la cruauté au prêtre et à l'assistant ambigu. Ce mélodrame placide et pervers d'un martyre idéalement scénarisé marque bien l'évolution de la mentalité religieuse du siècle qui accorde son certificat de décence à un "pompier " mais qui le déniait à Delacroix, vingt ans plus tôt.

Ce Martyre de saint Donatien et saint Rogatien devait être réalisé par Charles-Achille d'Hardiviller (1795-ap. 1835), peintre de la duchesse de Berry ${ }^{64}$ dont le séjour à Nantes en 1828 peut avoir déterminé ce choix. En 1831, Ch. Gabet mentionne « qu'il avait été 
chargé par le ministre de l'intérieur d'un tableau pour la ville de Nantes représentant le martyre de st. Donatien et de st. Rogatien ${ }^{65}$ » et plusieurs auteurs le citent ${ }^{66}$. Dans l'état actuel des recherches, il semble bien que la peinture de Vauchelet ait suppléé à l'absence de celle d'Hardiviller dont l'important œuvre gravé ne comporte rien sur ce thème ${ }^{67}$.

Figure 10

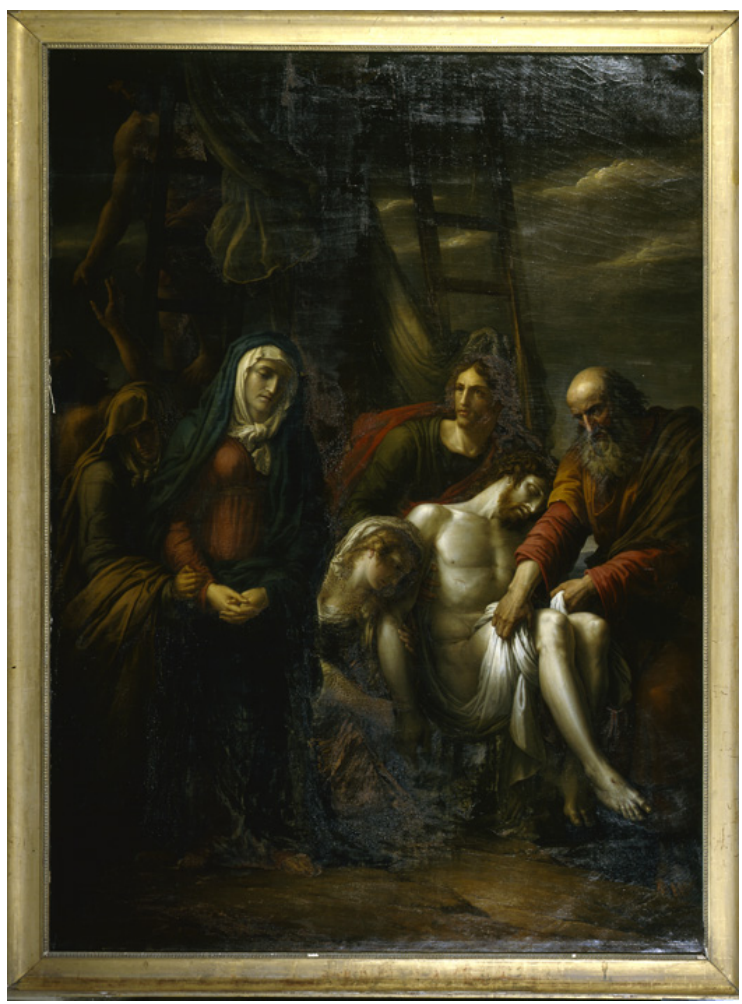

Étienne-Barthélémy Garnier. La Descente de Croix. Nantes, cathédrale Saint-Pierre.

Phot. D. Pillet. @ Région Pays de la Loire - Inventaire général, 8544 220VA.

Nous n'avons pas plus de certitudes quant au rôle de la duchesse de Berry dans le don d'autres tableaux à la cathédrale. Selon une lettre conservée aux Archives nationales ${ }^{68}$ que confirment A. Guépin et E. Bonamy ${ }^{69}$, elle aurait offert la grande Descente de Croix d'Étienne-Barthélémy Garnier (1759-1849) (fig. 10). Pourtant, une lettre du 9 juin 1828 du ministre de l'Intérieur au préfet explicite clairement sa provenance : « Mr le Baron, j'ai l'honneur de vous annoncer que je viens d'accorder à la cathédrale de Nantes le tableau de la Vierge mère de douleur ${ }^{70}$ exécuté par Garnier, membre de l'Académie Royale des Beaux-Arts $»^{71}$. Peinte à Paris pour l'autel de la Vierge de l'église de la Madeleine ${ }^{72}$, exposée au Salon de $1827^{73}$, cette Vierge, mère de douleur ne procède certes pas d'une demande locale. Ne peut-il s'agir d'une complaisance de l'administration envers la duchesse, pour lors en grâce, à qui l'on aurait délégué le prestige de ce don quand elle vint à Nantes, précisément durant l'été $1828^{74}$ ? Nulle inscription sur l'œuvre, nulle signature ${ }^{75}$ ne permet d'étoffer cette hypothèse mais la Vierge, mère de douleur de la cathédrale, inédite, s'accommode bien d'un surcroît de mystère. La stricte frise de la composition, l'éclairage crépusculaire et l'atonie des coloris donnent à la scène l'atmosphère étrange d'un drame antique revisité par l'esprit du romantisme. Les purs profils grecs, leur ligne sereine dans cette ambiance pesante, doivent beaucoup 
moins à Vien qui fut l'un des maîtres de Garnier qu'à Girodet ou Gérard dont ils rappellent la morbidesse. Dans son Salon de 1827, Jal attaque la vacuité d'une telle démonstration: "Pour les âmes tendues, ardentes, le tableau de M. Garnier ou un tableau de Raphaël, c'est tout un ; ce n'est pas tout un pour la majorité des chrétiens de ce temps-ci ; il est si peu de ces bonnes âmes $! »^{76}$. Très sévère, le critique n'ignore pas les limites de ce talent officiel ${ }^{77}$ mais néglige d'en souligner la solidité.

$\mathrm{Au}$ Martyre de saint Donatien et saint Rogatien de Vauchelet faisait face autrefois le Martyre de saint Gervais et saint Protais du Nantais Auguste Blondel (1799-1872). Copie réduite assez fidèle à l'original d'Eustache Le Sueur ${ }^{78}$, elle honore l'humble habileté d'un professeur de dessin de la ville, à l'opposé de la facilité bavarde de son compatriote Édouard Jolin (1817-ap. 1869). Né à Nantes mais formé à Paris par Paul Delaroche, Jolin expose dans son Martyre de saint Gohard sa conception d'une peinture d'histoire d'un dynamisme et d'une théâtralité de "peplum ». En 1845, Mgr de Hercé avait demandé au ministère de l'Intérieur un tableau du massacre du 24 juin 843 lors duquel périrent pendant la messe l'évêque Gohard et ses fidèles sous les coups des Normands ${ }^{79}$. Il suggérait aussi le nom de Jolin, «jeune artiste nantais », et qu'il représentât le vieux chœur roman qu'on s'apprêtait alors à démolir. Exposée à Paris au Salon de 1852 et à Nantes deux ans plus $\operatorname{tard}^{80}$, la toile de Jolin s'inscrit bien dans les recherches des années 1850, recherches d'effets neufs, spectaculaires et réalistes qui débouchent sur le pompiérisme de la seconde moitié du siècle ${ }^{81}$.

\section{Les artistes nantais}

41 La réorganisation du culte au XIX ${ }^{e}$ siècle et la remise en ordre de la cathédrale s'accompagnent de nombreuses interventions d'artistes locaux. Esprit du temps attaché à un certain retour aux ressources de la province, ou urgence des commandes à passer pour restituer sa dignité au sanctuaire, force est de constater la relative modestie des œuvres fournies par les peintres de la région. Tel A. Blondel, ceux-ci se voient surtout confier l'exécution de copies, à moins qu'une brillante carrière parisienne ne permette une participation plus prestigieuse comme pour E. Jolin ou J.-É. Delaunay.

Malgré sa présence à certains salons parisiens, F. Donné (?-1844) témoigne ainsi dans la première moitié du siècle du large écart qualitatif séparant la production nantaise et celle des peintres rodés à l'âpre émulation de la capitale. Sa Tempête apaisée, datée de 1838 et exposée au Salon de l'année suivante, ne franchit pas les bornes d'un romantisme assez timide : animés d'aucun souffle, les personnages s'épuisent l'âme en d'ostentatoires platitudes et la voile du navire claque en vain. Souvenir d'un vaste tableau d'Ary Scheffer (Saint Thomas d'Aquin prêchant pendant la tempête, Salon de 1824, Paris, musée du Petit Palais), la composition qui se veut héroïque reste désespérément conventionnelle. Plus maladroite encore, la grande figure d'Aaron (datée de 1834, pendant d'un Moïse par Sotta) ${ }^{82}$ confinerait à la caricature si l'on n'était assuré du conformisme des bonnes intentions du peintre. Cette honnête absence de talent s'exerce pleinement dans la copie du Baptême du Christ qu'il réalisa vers 1828 pour remplacer l'original d'Ottavio Vannini (1585-1644), installé dans la chapelle des fonts baptismaux à la fin de $1809^{83}$. Destinée au même emplacement, la réplique froide et à peine appliquée qu'en livre Donné dessine bien les limites que ses commanditaires assignaient à ses activités à la cathédrale. 
À la génération suivante, l'exemple moins artisanal de Vincent Vidal (1811-1887) illustre néanmoins cette même ambiguïté du rôle des artistes du cru dans le chantier de la cathédrale. Vidal, peintre d'origine méridionale vivant à Rennes et à Paris, possédait un manoir à Bannalec, "Kerlagadic ", et exposa plusieurs fois à Nantes ${ }^{84}$ des sujets bretons notamment dont il s'était fait une spécialité. De cet artiste mondain qui fréquentait les courtisans de l'Empire, qui portraitura Eugénie et dont Baudelaire fustigea "la folie de la propreté $»^{85}$, Saint-Pierre conserve deux copies d'après des maîtres italiens du XVII ${ }^{e}$ siècle, fait assez surprenant si l'on considère le domaine habituel de ses prédilections. La première réplique, Le Roi David jouant de la harpe d'après Le Dominiquin ${ }^{86}$, peut n'avoir constitué qu'une acquisition de circonstance. La seconde reproduit La Remise des clefs à saint Pierre de Guido Reni ${ }^{87}$, témoignage fort probable d'un sujet choisi par le chapitre de la cathédrale.

Figure 11

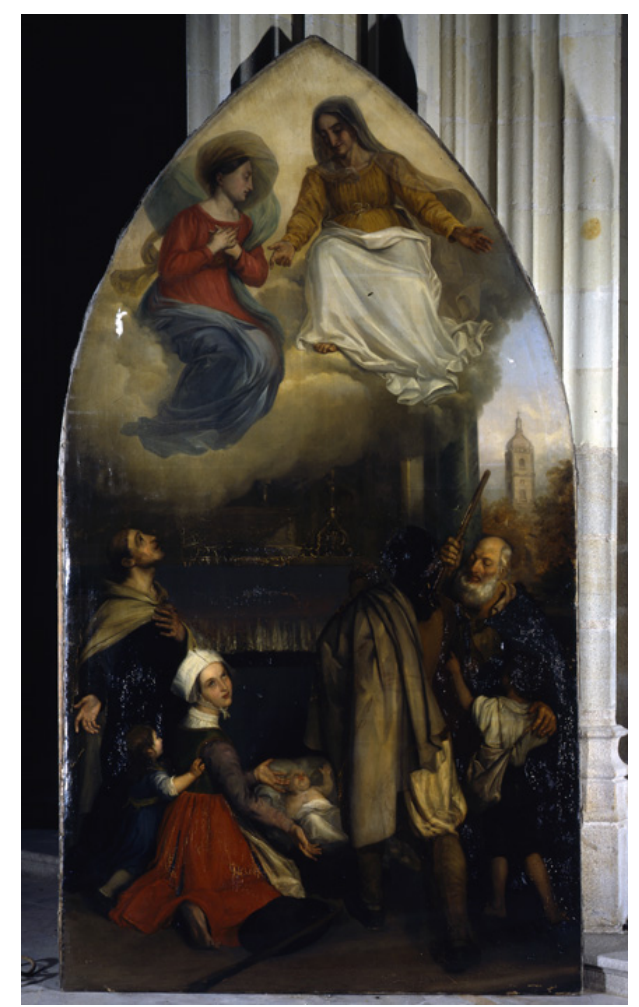

Vincent Vidal. Ex-voto. Nantes, cathédrale Saint-Pierre.

Phot. D. Pillet. @ Région Pays de la Loire - Inventaire général, 8544 215VA.

Plus important, le grand ex-voto ogival signé "Vidal $\mathrm{P}^{\mathrm{t}}$ Parisiis» (fig. 11), situe paradoxalement son auteur dans un contexte local que soulignent certains traits régionaux : type populaire des orants, pittoresque des costumes... Malheureusement abîmée et repeinte, et malgré de beaux morceaux, l'œuvre n'offre plus aujourd'hui qu'un intérêt historique en documentant un aspect souvent négligé de la dévotion au $\mathrm{XIX}^{\mathrm{e}}$ siècle. Selon un schéma traditionnel, la présence divine se situe au registre supérieur, dans une nuée où se trouvent la Vierge et sainte Anne. La scène figure-t-elle une apparition ou symbolise-t-elle une invocation? L'attitude des personnages de la zone inférieure ne permet pas de le préciser avec exactitude. Des paysans semblent avoir amené un nouveau-né à l'intérieur de l'église, sans doute à l'instigation du moine, 
sur la gauche de l'image. Bien que sainte Anne soit invoquée comme protectrice de l'enfance et de l'éducation, l'iconographie de cet ex-voto demeure floue, comme du reste la place exacte qu'il occupait dans l'édifice. Des recherches plus poussées sur les relations des sanctuaires de Nantes et de Sainte-Anne-d'Auray au XIX siècle apporteraient sans doute des précisions sur la commande de l'œuvre.

Les commandes réalisées par Joachim Sotta (1810-1877) pour la cathédrale dénotent une plus large confiance de la fabrique envers les qualités d'un artiste local. D'origine italienne, Sotta arriva en France en 1825 et après une formation à Paris auprès de Gros et de Hersent, s'établit à Nantes en 1833 ; il habitait rue du Château et professait à l'école de dessin de la ville. Il y consacra une large part de son activité à décrire la notabilité nantaise de son époque: effigies de Pierre Haudaudine ${ }^{88}$, de Frédéric Cailliaud $^{89}$, de Mgr de Hercé ${ }^{90}$ ou de Mgr Angebault, évêque d'Angers ${ }^{91}$, etc. Hors cette production commerciale, Sotta peignit quelques portraits plus intimes parmi lesquels son propre visage ${ }^{92}$ et celui de son jeune élève Delaunay ${ }^{93}$. La plus ancienne de ses œuvres conservées à la cathédrale est un Moïse qu'il réalisa vraisemblablement en 1834 en pendant d'un Aaron de Donné daté de la même année. La figure ne manque pas d'une certaine puissance et d'une volonté de monumentalité sans doute issues des ateliers qu'il avait fréquentés. Mais le grand œuvre de Sotta à Saint-Pierre devait orner la chapelle Saint-Félix où, dans les boiseries néo-gothiques conçues par Liberge, s'encastraient ses tableaux du Christ, des évangélistes et des prophètes séparés par des anges ${ }^{94}$. Les quatorze toiles qui viennent d'être retrouvées dans la crypte du XIX siècle sont pour l'instant en trop mauvais état de conservation pour être correctement étudiées. En 1865, on faisait encore appel au peintre pour la chapelle du Sacré-Cœur et du Saint-Sacrement, en souffrance d'ornement depuis quarante ans, et pour laquelle il travailla à une belle copie qu'on peut encore y voir. D'une sensibilité déjà saintsulpicienne, la composition reprend pourtant fidèlement un modèle du XVIII ${ }^{e}$ siècle, alors très diffusé par l'imagerie pieuse, œuvre de Pompeo Batoni (Rome, église du Gesù) ${ }^{95}$. Mais les qualités du Sacré-Cœur de Sotta pâlissent singulièrement, confrontées au chef-d'œuvre de son élève exposé dans la même chapelle. 


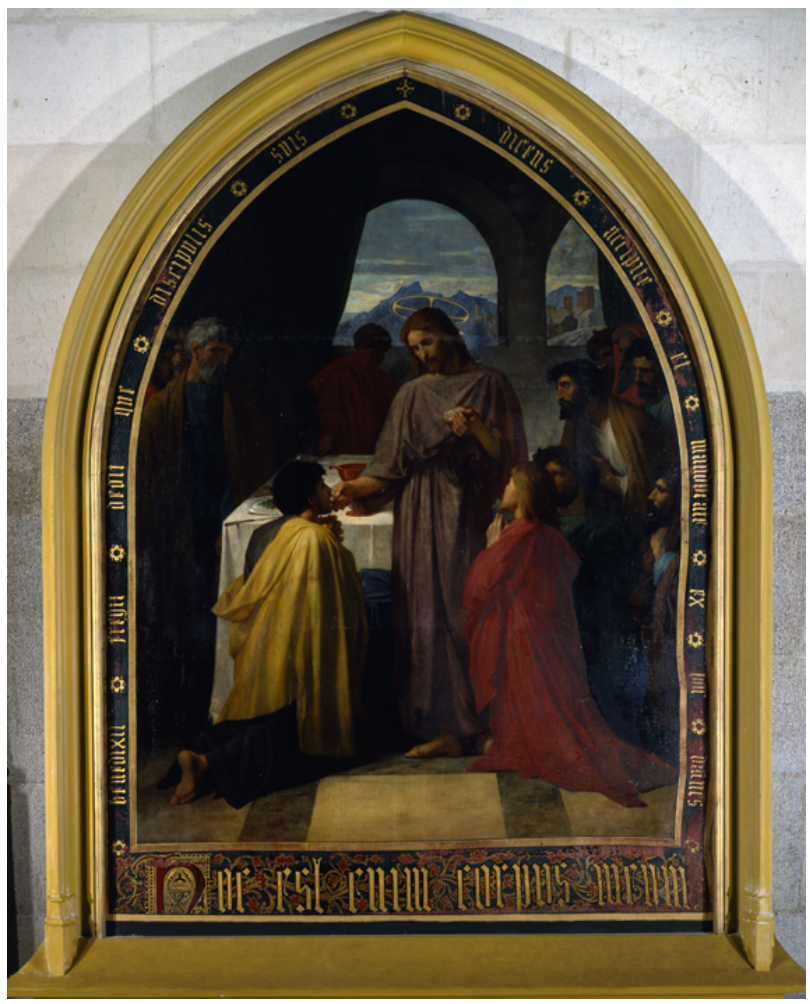

Jules-Élie Delaunay. La Communion des apôtres. Nantes, cathédrale Saint-Pierre.

Phot. D. Pillet. @ Région Pays de la Loire - Inventaire général, 8544 214VA.

La Communion des apôtres (fig. 12) de Jules-Élie Delaunay (1828-1891) éclipse le reste de la production locale et clôt brillamment le cycle des peintres nantais à Saint-Pierre. Simultanément, la position de l'artiste, d'abord élève de Sotta qui le confia par la suite à Flandrin, confère une place symbolique à son tableau, au crépuscule d'une époque dont l'esprit ne soufflera plus après lui. À Nantes, Delaunay donnera le meilleur de son talent de décorateur religieux à Saint-Nicolas (de 1855 à 1877) et à la chapelle du couvent de la Visitation (1863-1864), véritables sanctuaires de son $\operatorname{art}^{96}$. Quant à la Cène, nous ne reviendrons pas ici sur l'originalité du dessinateur ni sur sa façon d'aborder un thème rebattu par une approche personnelle et sensible, d'un intimisme et d'une sobriété dignes de Flandrin: la notice du catalogue de l'exposition consacrée à l'artiste en 1988-1989 les analyse parfaitement pour la version du musée des Beaux-Arts ${ }^{97}$. En effet, le tableau reprend une première composition exécutée à Rome en 1861 et achetée par l'État en 1864. La jugeant trop sombre pour la cathédrale, l'abbé Lepré, secrétaire de Mgr Jacquemet, en commanda une réplique que l'artiste dut totalement repenser pour répondre aux directives d'un clergé dont les exigences ne s'étaient pas émoussées en quarante ans. À Paris, Delaunay modifia donc profondément sa Communion des apôtres en lui donnant un format ogival qui en appuie le caractère médiéval, accentué encore par l'orle de l'inscription gothique qui la cerne. En élargissant le champ perspectif, en remaniant le dessin de la plupart des personnages et en éclaircissant considérablement sa palette, l'artiste subordonnait aussi l'intensité de la scène à sa lisibilité. Datée de 1862 , la toile de Delaunay est la dernière œuvre d'importance acquise pour le décor de la cathédrale, elle le ponctue en point d'orgue d'une note grave et parfaitement tenue. 
«Parlerai-je de peinture ? " maugréait A. Guépin en $1832^{98}$ à propos de la cathédrale, alors qu'aucun des bons tableaux que nous commentons ne s'y trouvait. Trente ans plus tard, la question ne se posera plus et aujourd'hui, il parait évident que Saint-Pierre de Nantes possède, après Paris mais peu d'autres grandes villes, l'un des ensembles de tableaux religieux du siècle dernier les plus cohérents de notre pays. Après l'autodafé du 20 novembre 1793 et la disparition des « hochets de la superstition ${ }^{99}$ ", les Nantais de la Restauration eurent à cœur de redonner à leur cathédrale un cadre digne de l'histoire du lieu. Précédant l'extension de l'édifice, ce nouveau décor souligne l'importance du rôle de l'image dans le culte revivifié du xix siècle et coïncide avec une renaissance de la grande peinture d'inspiration chrétienne.

\section{Annexe : répertoire des peintures de la cathédrale de Nantes}

\section{Anonyme}

\section{(début du XVIII ${ }^{\mathrm{e}}$ siècle)}

1 - Saint René

Peinture murale

Inscription : «S. René »

\section{Anonyme}

(début du XIX ${ }^{\mathrm{e}}$ siècle ?)

2 - La déploration sur le Christ mort (Piéta)

Huile sur toile : 2,84 x 1,90 m.

\section{Attribué à Jean-Louis Bézard}

(Toulouse, 1799-?, 1881)

3 - Deux anges portant le Saint Sacrement

Peinture murale

Bibliographie : DURVILLE, abbé Georges. Études sur le vieux Nantes d'après les documents originaux. Nantes : L. Durance, 1900-1915, p. CXXX-CXXXI (?)

\section{Auguste Blondel}

(Nantes, 1799-Nantes, 1872)

D’après Eustache Le Sueur (1616-1655)

4 - Saint Gervais et saint Protais conduits au martyre

Huile sur toile ; $2,50 \times 3,10 \mathrm{~m}$.

Bibliographie : GABORIT, abbé Prosper. Histoire de la cathédrale de Nantes. Nantes : impr. Bourgeois, 1888, p. 75 ; FURET, Jules, CAILLÉ, Dominique. «Les cathédrales de Nantes ». BSAN, 1905, p. 230 ; RUSSON, Jean-Baptiste, DURET, Dominique. La cathédrale de Nantes. Savenay : impr. Roumegoux et cie, 1933, p. 106.

\section{Mathurin (?) Bonnecamp}

( $1^{\mathrm{e}}$ moitié du XVII ${ }^{\mathrm{e}}$ siècle)

5 - L'adoration des Mages 
Huile sur toile ; $3,95 \times 2,65 \mathrm{~m}$.

Signature : «Bonnecamp pincit $\operatorname{In}[\mathrm{v}]$ enia $^{\mathrm{t}}$ »

Bibliographie: GUÉPIN, Ange, BONAMY, Eugène. Nantes au XIX siècle, statistique topographique, industrielle et morale. Nantes: P. Sebire, 1835, rééd. Nantes: Centre de recherche politique, université de Nantes, p. 115 ; DURVILLE, abbé Georges. Études sur le vieux Nantes d'après les documents originaux. Nantes : L. Durance, 1900-1915, p. CXXXVII ; RUSSON, Jean-Baptiste, DURET, Dominique. La cathédrale de Nantes. Savenay: impr. Roumegoux et cie, 1933, p. 108 ; AUZAS, Pierre-Marie. " Les œuvres d'art du diocèse de Nantes ». Mémoires de la Société d'histoire et d'archéologie de Bretagne, 1969, t. XLIX, p. 15-37, p. 29.

Archives : Nantes, Bibl. mun., fonds Boismen, ms. 3161, $\mathrm{n}^{\circ} 27$.

Jules-Élie Delaunay

\section{(Nantes, 1828-Paris, 1891)}

6 - La communion des apôtres

Huile sur toile ; 2,74 x 1,97 m.

Signature : « Elie Delaunay Paris 1862 »

Bibliographie : GABORIT, abbé Prosper. Histoire de la cathédrale de Nantes. Nantes : impr. Bourgeois, 1888, p. 75 ; FURET, Jules, CAILLÉ, Dominique. "Les cathédrales de Nantes ». BSAN, 1905, p. 229 ; G. Rondeau, 1931, p. 234 = RONDEAU, Gaétan. Nantes dans la France de l'Ouest. Paris: Plon, 1941 ; RUSSON, Jean-Baptiste, DURET, Dominique. La cathédrale de Nantes. Savenay: impr. Roumegoux et cie, 1933, p. 109; JULIA, Isabelle, DUPONT, Patrick. Jules-Élie Delaunay. Cat. expo., Nantes, musée des Beaux-Arts, septembrenovembre 1988, Paris, musée Hébert, janvier-mars 1989. Saint-Sébastien : ACL Crocus, 1988, p. 103-107, $\mathrm{n}^{\circ} 44$.

\section{Jean-Baptiste-François Désoria}

(Paris, 1758-Cambrai, 1832)

7 - Saint Charles-Borromée communiant les pestiférés

Huile sur toile ; $2,82 \times 1,87 \mathrm{~m}$.

Signature : « Des[oria] 1827 »

Bibliographie: GUÉPIN, Ange, BONAMY, Eugène. Nantes au XIX siècle, statistique topographique, industrielle et morale. Nantes: P. Sebire, 1835, rééd. Nantes : Centre de recherche politique, université de Nantes, 1981, p. 115 ; GABORIT, abbé Prosper. Histoire de la cathédrale de Nantes. Nantes : impr. Bourgeois, 1888, p. 75 ; FURET, Jules, CAILLÉ, Dominique. "Les cathédrales de Nantes ». BSAN, 1905, p. 229 ; DURVILLE, abbé Georges. Études sur le vieux Nantes d'après les documents originaux. Nantes : L. Durance, 1900-1915, p. CXXXVI; RUSSON, Jean-Baptiste, DURET, Dominique. La cathédrale de Nantes. Savenay: impr. Roumegoux et cie, 1933, p. 107 ; FOUCART, Bruno. Le renouveau de la peinture religieuse en France (1800-1860). Paris : Arthena, 1987, p. 401.

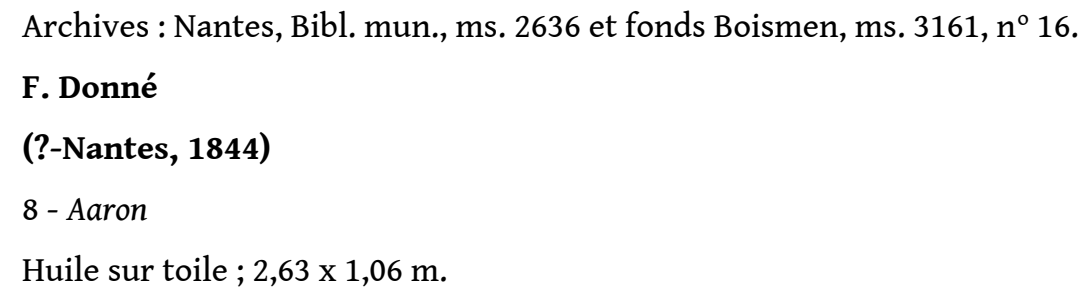


Signature : « $1834 \mathrm{~F}$. Donné $\mathrm{F}^{\mathrm{t}}$ »

Bibliographie : DURVILLE, abbé Georges. Études sur le vieux Nantes d'après les documents originaux. Nantes : L. Durance, 1900-1915, p. CXXXVII ; RUSSON, Jean-Baptiste, DURET, Dominique. La cathédrale de Nantes. Savenay : impr. Roumegoux et cie, 1933, p. 108.

9 - La tempête apaisée

Huile sur toile ; $3,24 \times 2,51 \mathrm{~m}$.

Signature : «F. Donné 1838 »

Bibliographie: RUSSON, Jean-Baptiste, DURET, Dominique. La cathédrale de Nantes. Savenay : impr. Roumegoux et cie, 1933, p. 108 ; FOUCART, Bruno. Le renouveau de la peinture religieuse en France (1800-1860). Paris : Arthena, 1987, p. 401.

Archives : Nantes, Bibl. mun., fonds Boismen, ms. 3161, $\mathrm{n}^{\circ} 1$.

- D’après Ottavio Vannini (1585-1644)

10 - Le baptême du Christ

Huile sur toile ; $3,16 \times 2,08 \mathrm{~m}$.

Bibliographie: GUÉPIN, Ange, BONAMY, Eugène. Nantes au XIX siècle, statistique topographique, industrielle et morale. Nantes: P. Sebire, 1835, rééd. Nantes: Centre de recherche politique, université de Nantes, 1981, p. 114-115 ; GABORIT, abbé Prosper. Histoire de la cathédrale de Nantes. Nantes : impr. Bourgeois, 1888, p. 75 ; FURET, Jules, CAILLÉ, Dominique. "Les cathédrales de Nantes ». BSAN, 1905, p. 229 ; DURVILLE, abbé Georges. Études sur le vieux Nantes d'après les documents originaux. Nantes : L. Durance, 1900-1915, p. CXXXVI ; RUSSON, Jean-Baptiste, DURET, Dominique. La cathédrale de Nantes. Savenay : impr. Roumegoux et cie, 1933, p. 106.

Archives : Nantes, Bibl. mun., fonds Boismen, ms. 3161, $\mathrm{n}^{\circ} 15$.

Charles Doussault

(Fougères, 1806-?, ap. 1873)

11 - La Vierge à l'Enfant avec trois anges

Huile sur toile ; $3,05 \times 2,20 \mathrm{~m}$.

Signature : «C. Doussault $1838 »$

Bibliographie: RUSSON, Jean-Baptiste, DURET, Dominique. La cathédrale de Nantes. Savenay : impr. Roumegoux et cie, 1933, p. 106.

Archives : Nantes, Bibl. mun., fonds Boismen, ms. 3161, $\mathrm{n}^{\circ} 13$.

\section{Charles Errard}

(Bressuire, 1570-?, av. 1630)

12 - La remise des clefs à saint Pierre

Huile sur toile ; $2,84 \times 2,14 \mathrm{~m}$.

Signature : « Errard»

Bibliographie: GUÉPIN, Ange, BONAMY, Eugène. Nantes au XIX siècle, statistique topographique, industrielle et morale. Nantes: P. Sebire, 1835, rééd. Nantes : Centre de recherche politique, université de Nantes, 1981, p. 112; CHENNEVIÈRES-POINTEL, Charles-Philippe de. Recherches sur la vie et les ouvrages de quelques peintres provinciaux de l'ancienne France. Paris : Dumoulin, 1847-62, t. 3, p. 57-72 et 292-300 ; FILLON, Benjamin. Documents sur Charles Errard : peintre et architecte. Nantes : impr. A. Guérand et Cie, 1853, 
p. 19-32 ; BOISMEN, E. La vérité sur la paternité des peintures de la coupole et du vieux chœur roman de la cathédrale de Nantes. Vannes : E. Lafolye impr., 1890, p. 9-12 ; DURVILLE, abbé Georges. Études sur le vieux Nantes d'après les documents originaux. Nantes: L. Durance, 1900-1915, p. CXXXVII ; RUSSON, Jean-Baptiste, DURET, Dominique. La cathédrale de Nantes. Savenay: impr. Roumegoux et cie, 1933, p. 107 ; AUZAS, Pierre-Marie. «Les œuvres d'art du diocèse de Nantes ». Mémoires de la Société d'histoire et d'archéologie de Bretagne, 1969, t. XLIX, p. 15-37, p. 30.

Archives : Nantes, Bibl. mun., fonds Boismen, ms. 3161, $\mathrm{n}^{\circ} 18$. impr. Vve Camille Mellinet, 1845, p. 7 ; FLANDRIN, Hippolyte. Lettres et pensées d'Hippolyte Flandrin. Éd. Henri Delaborde. Paris : H. Plon, 1865, p. 246 ; GABORIT, abbé Prosper. Histoire de la cathédrale de Nantes. Nantes: impr. Bourgeois, 1888, p. 74-75; FURET, Jules, CAILLÉ, Dominique. «Les cathédrales de Nantes ». BSAN, 1905, p. 229 ; FLANDRIN, Louis. Un peintre chrétien au XIXe siècle. Hippolyte Flandrin. Paris: Perrin, 1909, p. 64-65; DURVILLE, abbé Georges. Études sur le vieux Nantes d'après les documents originaux. Nantes : L. Durance, 1900-1915, p. CXXX-CXXXI ; G. Rondeau, 1931, p. 234 ; RUSSON, Jean-Baptiste, DURET, Dominique. La cathédrale de Nantes. Savenay: impr. Roumegoux et cie, 1933, p. 104-105; AUZAS, Pierre-Marie. « Les œuvres d'art du diocèse de Nantes ». Mémoires de la Société d'histoire et d'archéologie de Bretagne, 1969, t. XLIX, p. 15-37, p. 30-31 ; FOUCART, Jacques, FOUCART, Bruno. Hippolyte, Auguste et Paul Flandrin, une fraternité picturale au XIX siècle. Cat. expo. Paris, musée du Luxembourg, novembre1984-février 1985, Lyon, musée des Beaux-Arts, mars-mai 1985. Paris: Réunion des musées nationaux, 1984, p. 66-69, $\mathrm{n}^{\text {os }} 11-12-13$; FOUCART, Bruno. Le renouveau de la peinture religieuse en France (1800-1860). Paris : Arthena, 1987, p. 88, 401. 


\section{Édouard Jolin}

Huile sur toile ; $2,06 \times 1,37 \mathrm{~m}$.

Signature : « Ed. Hauser f $\mathrm{fct}^{\mathrm{ct}} 1838$ »

Bibliographie: RUSSON, Jean-Baptiste, DURET, Dominique. La cathédrale de Nantes. Savenay : impr. Roumegoux et cie, 1933, p. 106 ; FOUCART, Bruno. Le renouveau de la peinture religieuse en France (1800-1860). Paris : Arthena, 1987, p. 401.

\section{Attribué à Charles-François Jalabert}

(Nîmes, 1819-Paris, 1901)

ou L.-E. de La Boëssière (?)

16 - Sainte Philomène adorée par deux anges

Huile sur toile; $1,98 \times 1,45 \mathrm{~m}$.

Signature : «L E de la Boëssière 1858 »

Bibliographie: La Notice sur la chapelle Saint-Clair dans l'église cathédrale de Nantes. Nantes: impr. Vve Camille Mellinet, 1845, mentionne une Sainte Philomène dans la chapelle Saint-Clair. La même année, les archives évoquent le peintre Jalabert pour cette commande (Arch. dép. Loire-Atlantique, Arch. dioc. : 125 J 306) ; G. Rondeau, 1941, p. 234: mentionne Boissier (André-Claude Boissier, Nantes, 1760-Château-Gontier, 1833) sans préciser aucun titre d'œuvre ; RUSSON, Jean-Baptiste, DURET, Dominique. La cathédrale de Nantes. Savenay : impr. Roumegoux et cie, 1933, p. 106-107 : situent l'œuvre dans la chapelle des fonts baptismaux.

(Nantes, 1817-?, ap. 1869)

17 - Le martyre de saint Gohard

Huile sur toile ; $3,90 \times 2,88 \mathrm{~m}$.

Signature : « 1852 E. Joli(n)»

Bibliographie : GABORIT, abbé Prosper. Histoire de la cathédrale de Nantes. Nantes : impr. Bourgeois, 1888, p. 75 ; FURET, Jules, CAILLÉ, Dominique. «Les cathédrales de Nantes ». BSAN, 1905, p. 229-230, n.1 ; DURVILLE, abbé Georges. Études sur le vieux Nantes d'après les documents originaux. Nantes : L. Durance, 1900-1915, p. CXXXVI ; RUSSON, Jean-Baptiste, DURET, Dominique. La cathédrale de Nantes. Savenay : impr. Roumegoux et cie, 1933, p. 107 ; FOUCART, Bruno. Le renouveau de la peinture religieuse en France (1800-1860). Paris : Arthena, 1987, p. 402.

Archives : Arch. nat. : $\mathrm{F}^{19} 7771$

\section{Atelier de Charles de La Fosse}

(Paris, 1636-Paris, 1716)

18 - La crucifixion

Huile sur toile ; $1,90 \times 0,98 \mathrm{~m}$. (sans l'agrandissement)

Autrefois dans le retable de Tugal Caris dans la chapelle Notre-Dame-de-Pitié, restauré et replacé en 1991.

Bibliographie : GABORIT, abbé Prosper. Histoire de la cathédrale de Nantes. Nantes : impr. Bourgeois, 1888, p. 75 ; FURET, Jules, CAILLÉ, Dominique. « Les cathédrales de Nantes ». BSAN, 1905, p. 229 ; RUSSON, Jean-Baptiste, DURET, Dominique. La cathédrale de Nantes. Savenay : impr. Roumegoux et cie, 1933, p. 111 ; AUZAS, Pierre-Marie. «Les œuvres 
d'art du diocèse de Nantes ». Mémoires de la Société d'histoire et d'archéologie de Bretagne, 1969, t. XLIX, p. 15-37, p. 29.

Archives : Nantes, Bibl. mun., fonds Boismen, ms. 3161, $\mathrm{n}^{\circ} 10$.

\section{Jules Laure}

(Grenoble, 1806-Paris, 1861)

19 - Le Christ

Disparu

20 - La Vierge

Huile sur toile ; $2,19 \times 0,48 \mathrm{~m}$.

21 - Saint Jean

Huile sur toile ; $2,19 \times 0,48 \mathrm{~m}$.

22 - Saint Léonard

Disparu

23 - Saint Laurent

Huile sur toile ; $1,44 \times 0,66 \mathrm{~m}$.

24 - Saint Vincent

Huile sur toile ; $1,44 \times 0,66 \mathrm{~m}$.

25 - Sainte Radegonde

Disparu

26 - Sainte Madeleine

Disparu

27 - Saint Denis

Huile sur toile : $1,44 \times 0,66 \mathrm{~m}$.

Signature : «Jules Laure »

Autrefois insérés dans les boiseries de la chapelle Saint-Clair, retrouvés en 1991.

Bibliographie: Notice sur la chapelle Saint-Clair dans l'église cathédrale de Nantes. Nantes: impr. Vve Camille Mellinet, 1845, p. 9-10 ; RUSSON, Jean-Baptiste, DURET, Dominique. La cathédrale de Nantes. Savenay : impr. Roumegoux et cie, 1933, p. 106.

\section{François Le Moyne}

(Paris, 1688-Paris, 1737)

28 - Saint Jean-Baptiste

Huile sur toile ; $3,25 \times 2,12 \mathrm{~m}$.

Signature : «F. Lemoyne F. 1715 »

Bibliographie: GUÉPIN, Ange, BONAMY, Eugène. Nantes au XIX siècle, statistique topographique, industrielle et morale. Nantes : P. Sebire, 1835 , rééd. Nantes : Centre de recherche politique, université de Nantes, 1981, p. 115; GABORIT, abbé Prosper. Histoire de la cathédrale de Nantes. Nantes : impr. Bourgeois, 1888, p. 75 ; FURET, Jules, CAILLÉ, Dominique. «Les cathédrales de Nantes ». BSAN, 1905, p. 229 ; DURVILLE, abbé Georges. Études sur le vieux Nantes d'après les documents originaux. Nantes: L. Durance, 1900-1915, p. CXXXVII ; G. Rondeau, 1941, p. 234 ; RUSSON, Jean-Baptiste, DURET, Dominique. La cathédrale de Nantes. Savenay : impr. Roumegoux et cie, 1933, p. 
108 ; BORDEAUX, Jean-Luc. François Le Moyne and his generation, 1688-1737. Paris : Arthena, 1984, p. 70-71, nº 4 .

\section{D'après Carlo Maratti}

(Camerano, 1625-Rome, 1713)

29 - L'apparition de la Vierge à saint Philippe Néri

Huile sur toile ; $2,16 \times 1,47 \mathrm{~m}$.

Déposé à la cathédrale en 1966, le tableau a été restitué au musée des Beaux-Arts en 1991 (cat. $1859, \mathrm{n}^{\circ} 418$; cat. $1876, \mathrm{n}^{\circ} 110$; cat. 1883 , p. 87 ; cat. $1903, \mathrm{n}^{\circ} 299$; cat. $1913, \mathrm{n}^{\circ}$ 101 ; cat. $\left.1953, \mathrm{n}^{\circ} 101\right)$.

\section{Jean-Baptiste Mauzaisse}

\section{(Corbeil, 1784-Paris, 1844)}

30 - Saint Clair guérissant un aveugle

Huile sur toile ; $2,83 \times 1,86 \mathrm{~m}$.

Signature : « Mauzaisse 1828 »

Bibliographie: LACAMBRE, Geneviève et Jean. «La politique d'acquisition sous la Restauration : les tableaux d'histoire ». Bulletin de la Société de l'art français, 1972, p. 331 ; FOUCART, Bruno. Le renouveau de la peinture religieuse en France (1800-1860). Paris: Arthena, 1987, p. 402.

Archives : Arch. nat. : $\mathrm{F}^{21} 4$; Nantes, Bibl. mun., ms. 2636 et fonds Boismen, ms. 3161, $\mathrm{n}^{\circ}$ 3.

\section{Joachim Sotta}

(Malesco, 1810-Treillères, 1877)

31 - Moïse

Huile sur toile ; 2,63 x 1,07 m.

Bibliographie : DURVILLE, abbé Georges. Études sur le vieux Nantes d'après les documents originaux. Nantes : L. Durance, 1900-1915, p. CXXXVII ; RUSSON, Jean-Baptiste, DURET, Dominique. La cathédrale de Nantes. Savenay : impr. Roumegoux et cie, 1933, p. 108.

32 - Le Christ à la colonne

33 - La Vierge en pamoison

34 - Une sainte (?)

35 - Saint Jean Évangéliste

36 - Saint Matthieu

37 - Saint Marc

38 - Saint Luc

39 - Un prophète

40 - Un prophète

41 - Un prophète

42 - Un prophète

43 - Un prophète

44 - Un ange 
45 - Un ange

Huiles sur toile ; 0,28 x 1,06 m.

Bibliographie : RUSSON, Jean-Baptiste, DURET, Dominique. La cathédrale de Nantes. Savenay : impr. Roumegoux et cie, 1933, p. 106.

Ces quatorze tableaux, retrouvés en 1991, étaient autrefois insérés dans les boiseries de la chapelle Saint-Félix. L'emplacement des deux suivants n'est pas connu avec certitude :

46 - La Vierge

Huile sur toile ; $1,73 \times 0,46 \mathrm{~m}$.

47 - Sainte Marie l'Égyptienne (?)

Huile sur toile; $1,73 \times 0,46 \mathrm{~m}$.

Signature : «J. Sotta »

D’après Pompeo Batoni (1708-1787)

48 - Le Sacré-Cour

Huile sur toile ; 0,76 x 0,65 m.

Signature : «J. Sotta 1865 copie »

Bibliographie: RUSSON, Jean-Baptiste, DURET, Dominique. La cathédrale de Nantes. Savenay : impr. Roumegoux et cie, 1933, p. 109.

\section{Charles-Auguste Van Den Berghe}

(Beauvais, 1798 - Paris, 1853)

49 - L'ensevelissement de la Vierge

Huile sur toile ; 3,25 x 4,06 m.

Signature : " Aug $^{\text {te }}$ Vandenberghe 1835 »

Bibliographie: DANJOU, Pierre-Nicolas. «Notice sur Charles-Auguste Van den Berghe... ». Mémoires de la Société académique d'archéologie, sciences et arts du département de l'Oise, 1854, p. 358-360 ; GABORIT, abbé Prosper. Histoire de la cathédrale de Nantes. Nantes: impr. Bourgeois, 1888, p. 75 ; FURET, Jules, CAILLÉ, Dominique. «Les cathédrales de Nantes ». BSAN, 1905, p. 230 ; DURVILLE, abbé Georges. Études sur le vieux Nantes d'après les documents originaux. Nantes: L. Durance, 1900-1915, p. CXXXVII ; RUSSON, Jean-Baptiste, DURET, Dominique. La cathédrale de Nantes. Savenay: impr. Roumegoux et cie, 1933, p. 108 ; AUZAS, Pierre-Marie. "Les œuvres d'art du diocèse de Nantes ». Mémoires de la Société d'histoire et d'archéologie de Bretagne, 1969, t. XLIX, p. 15-37, p. 31 ; FOUCART, Bruno. Le renouveau de la peinture religieuse en France (1800-1860). Paris : Arthena, 1987, p. 402.

Archives : Arch. dép. Loire-Atlantique, 34 V1

Théophile-Auguste Vauchelet

(Passy, 1802 - ?, 1873)

50 - Le martyre de saint Donatien et saint Rogatien

Huile sur toile; $4,10 \times 2,64 \mathrm{~m}$.

Signature : « Vauchelet 1839 » 


\section{Tableaux disparus ou déplacés}

\section{Vincent Vidal}

(Carcassonne, 1811 - Paris, 1887)

51 - Ex-voto de sainte Anne

Huile sur toile ; 3,55 x 1,95 m.

Signature : «Vidal Pt Parisiis anno 18[35] »

52 - Le roi David jouant de la harpe

Huile sur toile ; 2,60 x 1,98 m.

D’après Guido Reni (1575-1642)

53 - La remise des clefs à saint Pierre

Huile sur toile ; 2,57 x 1,90 m.

Signature : illisible

\section{Anonyme}

(avant 1845)

54 - Saint Clair

Autrefois dans le transept nord. d'intérêt ».

\section{D'après Coypel (?)}

55 - Le lavement des pieds

Bibliographie : GABORIT, abbé Prosper. Histoire de la cathédrale de Nantes. Nantes : impr. Bourgeois, 1888, p. 75 ; FURET, Jules, CAILLÉ, Dominique. « Les cathédrales de Nantes ». BSAN, 1905, p. 229 ; RUSSON, Jean-Baptiste, DURET, Dominique. La cathédrale de Nantes. Savenay : impr. Roumegoux et cie, 1933, p. 106 ; RUSSON, Jean-Baptiste. La Passion des enfants nantais. Nantes: Impr. de l'Éclaireur de l'Ouest, 1945, p. 124 ; FOUCART, Bruno. Le renouveau de la peinture religieuse en France (1800-1860). Paris : Arthena, 1987, p. 402.

Archives : Arch. nat, $\mathrm{F}^{19} 7771$; Nantes, Bibl. mun., fonds Boismen, ms. 3161, $\mathrm{n}^{\circ} 17$.

Archives : Nantes, Bibl. mun., fonds Boismen, ms. 3161, nº 12.

D'après Domenico Zampieri dit Le Dominiquin (1581-1641)

Bibliographie: DURVILLE, abbé Georges. Études sur le vieux Nantes d'après les documents originaux. Nantes : L. Durance, 1900-1915, p. CXXXVII ; RUSSON, Jean-Baptiste, DURET, Dominique. La cathédrale de Nantes. Savenay : impr. Roumegoux et cie, 1933, p. 108.

Archives : Nantes, Bibl. mun., fonds Boismen, ms. 3161, $\mathrm{n}^{\circ} 23$.

Bibliographie: RUSSON, Jean-Baptiste, DURET, Dominique. La cathédrale de Nantes. Savenay : impr. Roumegoux et cie, 1933, p. 108 : « d'après Poussin ».

Archives : Nantes, Bibl. mun., fonds Boismen, ms. 3161, nº 19.

Bibliographie : Notice sur la chapelle Saint-Clair dans l'église cathédrale de Nantes. Nantes: impr. Vve Camille Mellinet, 1845, p. 3-4: «... une sorte de peinture appliquée sur la muraille nue. On aperçoit encore cette peinture noirâtre dans la seconde ogive du bras de croix, du côté de la sacristie»; RUSSON, Jean-Baptiste, DURET, Dominique. La cathédrale de Nantes. Savenay: impr. Roumegoux et cie, 1933, p. 108: «de peu 
Autrefois dans la chapelle Notre-Dame-de-Pitié

Signature : « H.C.J. Bel... 1729 »

Bibliographie: GABORIT, abbé Prosper. Histoire de la cathédrale de Nantes. Nantes: impr. Bourgeois, 1888, p. 75 ; FURET, Jules, CAILLÉ, Dominique. «Les cathédrales de Nantes ». BSAN, 1905, p. 230 ; RUSSON, Jean-Baptiste, DURET, Dominique. La cathédrale de Nantes. Savenay: impr. Roumegoux et cie, 1933, p. 111: «d'allure quelque peu fastueuse et solennelle ».

Archives : Nantes, Bibl. mun., fonds Boismen, ms. 3161, nº 20.

Eugène Delacroix

(Charenton-Saint-Maurice, 1798 - Paris, 1863)

56 - La Vierge du Sacré-Cœur

Huile sur toile ; 2,58 x 1,52 m.

Ajaccio, cathédrale

Bibliographie: DURVILLE, abbé Georges. Études sur le vieux Nantes d'après les documents originaux. Nantes: L. Durance, 1900-1915, p. CXXX-CXXXI ; SÉRULLAZ, Maurice. Mémorial de l'exposition Eugène Delacroix organisée au musée du Louvre. Paris : Musées nationaux, 1963, $\mathrm{n}^{\circ} 6$; JOHNSON, Lee. The Paintings of Eugène Delacroix : a critical catalogue. Oxford: Clarendon press, 1981-1989, vol. I, p. 163-165, $\mathrm{n}^{\circ} 153$; SÉRULLAZ, Maurice. Delacroix. Paris : Fayard, 1989, p. 69-70.

\section{Jean JALABERT}

$(-)$

57- La Visitation

d'après Sebastiano del Piombo

Huile sur toile?; dimensions inconnues.

Louis-François Loysel

(Paris, 1740 - Nantes, 1780)

58 - Le portail central de la cathédrale (?)

Autrefois dans la sacristie, signé et daté de 1775.

Bibliographie: RUSSON, Jean-Baptiste, DURET, Dominique. La cathédrale de Nantes. Savenay: impr. Roumegoux et cie, 1933, p. 76: "l'image [de la Vierge] se dressait devant le trumeau entourée des statues des apôtres ».

Le fonds Boismen de la bibliothèque municipale de Nantes conserve un devis manuscrit (ms. 3161) concernant la restauration de certains des tableaux de la cathédrale. Datable de la seconde moitié du xix ${ }^{\mathrm{e}}$ siècle, ce document fournit la description succincte de vingt-neuf peintures dont nous extrayons onze numéros correspondant à des œuvres disparues :

59 - « 2 - Portrait de $\mathrm{S}^{\mathrm{t}}$ Charles Boromée. $190^{c}$ sur $140^{c}$. Réentoilage et restauration. 600 f. ».

60 - « 4 - St Gérôme, par Quintin Mathys (sic). 100 sur 128. Parquetage et restauration. $700 \mathrm{f}$. Urgent $»$.

61 - « 5 - Le Denier de $S^{t}$ Pierre, école de Rubens. $85^{c}-115^{c}$. Restauration. 150 f. ».

62 - « 6 - L'adoration des bergers par Franck. $43^{\mathrm{c}}$ sur $33^{\mathrm{c}}$. Restauration. $80 \mathrm{f}$. ». 
63 - « 7 - Vierge à l'enfant, d'après Sasso Feratto (sic). $70^{c} .56^{c}$. Restauration. 40 f. ».

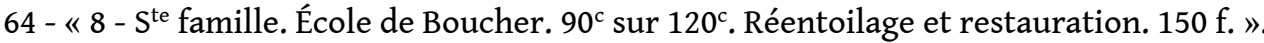

65 - « 9 - Le Christ chez Marthe et Marie. École du Poussin. $80^{c}$ sur $100^{c}$. Enlevage et restauration. $300 \mathrm{f}$. Urgent ».

66 - « 14 - $S^{t}$ Vincent de Paul prêchant. Par... $1830\left(200^{c}\right.$ sur $\left.160^{c}\right)$. Restauration. 100 f. ».

67 - « 21 - La Vierge de $S^{t}$ Luc. Nettoyage et vernis. 40 f. ».

68 - «22- $S^{t}$ Donatien et $S^{t}$ Rogatien. École Louis XV $\left(200^{c}\right.$ sur $\left.300^{c}\right)$. Restauration. 200

f. ».

69 - « 24 - Deux grands prêtres. 25 - Idem. 26 - Le Christ en croix. Nettoyage et vernis, les trois, $100 \mathrm{f} . »$.

\section{NOTES}

1. - L'Itinéraire de Bretagne en 1636... de Dubuisson-Aubenay évoque plus les tombeaux et les reliquaires de la cathédrale que son mobilier. Quant à la «visite pastorale» de 1638 qui inventorie le mobilier des églises de Nantes, nous n'en avons pas trouvé d'autre trace que sa mention (Bull. Soc. Archéol. Nantes (BSAN), n ${ }^{\text {os } 95-98 ~(1956-59), ~ 1958, ~ p . ~ X I I I) . ~}$

2. - GABORIT, abbé Prosper. Histoire de la cathédrale de Nantes. Nantes: impr. Bourgeois, 1888, p. 75 ; FURET, Jules, CAILLÉ, Dominique. «Les cathédrales de Nantes». BSAN, 1905, p. 229 ; RUSSON, Jean-Baptiste, DURET, Dominique. La cathédrale de Nantes. Savenay : impr. Roumegoux et cie, 1933, p. 111.

3. - Les Archives photographiques possèdent un cliché du retable avec la Crucifixion mais dans sa documentation sur les objets mobiliers, le service des Monuments historiques conserve deux fiches qui concernent sans doute notre tableau (classé le 5 novembre 1912) et qui établissent certaines confusions avec une petite Crucifixion entourée de saints et de deux donateurs peinte par Nautré en 1610 et conservée à l'église Saint-Similien de Nantes.

4. - Conservé au Palazzo Pitti de Florence. Il en existe d'autres versions d'atelier, telle celle vendue chez Sotheby le 10 décembre 1975 (cat. $\mathrm{n}^{\circ} 136 ; 0,68 \times 0,50 \mathrm{~m}$.). La composition s'inspire nettement de la partie supérieure de l'Ex-voto de la peste de Guido Reni (Bologne, Pinacoteca Nazionale).

5. - Lettre du maire au conservateur du 11 mai 1966, archivée dans le dossier de l'œuvre au musée des Beaux-Arts de Nantes, concernant ce dépôt demandé par Mgr Villepelet.

6. - Par exemple celle du musée des Beaux-Arts d'Orléans; voir O'NEILL, Mary. Les peintures de l'école française des XVII' et XVIII siècles. Orléans : musée des Beaux-Arts, 1981, 2 vol., v. 1, nº 83, repr. v. 2.

7. - Les données sur la biographie du peintre proviennent principalement de FILLON, Benjamin. Documents sur Charles Errard : peintre et architecte. Nantes : impr. A. Guérand et Cie, 1853, p. 19-32; CHENNEVIÈRES-POINTEL, Charles-Philippe de. Recherches sur la vie et les ouvrages de quelques peintres provinciaux de l'ancienne France. Paris: Dumoulin, 1847-62, 4 vol., tome III, p. 57-72 et 292-300 ; BOISMEN, E.. La vérité sur la paternité des peintures de la coupole et du vieux choeur roman de la cathédrale de Nantes. Vannes : E. Lafolye impr., 1890.

8. - RUSSON, J.-B., DURET, D., op. cit., p. 107. 
9. - Voir LENIAUD, Jean-Michel. «L'achèvement de la cathédrale de Nantes ». BSAN, t. 123, 1987, p. 229-279, ici p. 232.

10. - Ce relevé est signalé par BOISMEN, E., op. cit., p. 19, et MAILLARD, Émile. L'art à Nantes au XIX ${ }^{e}$ siècle. Paris : Libr. des impr. réunis, 1888, p. 14 et erratum p. 335.

11. - CHARAVAY, Étienne. Inventaire des autographes et des documents historiques composant la collection de M. Benjamin Fillon. Paris : Charavay frères, F. Naylor, 1879, série IX, p. 22, n 1620 , et Bulletin de la Maison Charavay (Paris, 3 rue de Furstenberg), février 1881, $\mathrm{n}^{\circ} 771$, p. 14, $\mathrm{n}^{\circ} 38.868$.

12. - Repr. dans PRÉAUD, Maxime. Mélancolies. Paris : Herscher, 1982, p. 114.

13. - Voir THUILLIER, Jacques. «Propositions pour Charles Errard, peintre ». Revue de l'Art, 1978, $\mathrm{n}^{\circ} 40 / 41$, p. $151-172$.

14. - GRANGES de SURGÈRES, Anatole Louis Théodore Marie de. Les artistes nantais... Paris: Charavay frères, s.d. [1898], p. 62.

15. - Nous songeons à l'Adoration des mages de Claude Vignon (1593-1670) conservée au Birmingham Museum of Art (Alabama) et à celle qu'on attribue parfois à Georges Lallemant (v. 1575-1636) conservée au musée des Beaux-Arts de Lille.

16. - Voir Autour de la Nativité dans la peinture des collections angevines. Cat. expo., Angers, 1989-90. Angers : Association culturelle du département de Maine-et-Loire, 1989, p. 118-119, nº 22.

17. - RUSSON, J.-B., DURET, D., op. cit., p. 108.

18. - BORDEAUX, Jean-Luc. François Le Moyne and his generation, 1688-1737. Paris : Arthena, 1984, p. $70-71, \mathrm{n}^{\circ} 4$, précise qu'il ne peut s'agir du Saint Jean-Baptiste de Saint-Eustache à Paris (1726), toujours in situ.

19. - En l'absence du tableau, nous ne proposons aucun nom pour l'auteur du modèle qui ne se situe peut-être pas dans l'orbite des Coypel. De plus, l'œuvre portait cette signature : «H.C.J. Bel[...] $1729 »$ (Bibl. mun. Nantes : Ms. 3161).

20. - RUSSON, J.-B., DURET, D., op. cit., p. 111.

21. - FOUCART, Bruno. Le renouveau de la peinture religieuse en France (1800-1860). Paris : Arthena, 1987.

22. - DELACROIX, Eugène. Journal. Champrosay, 17 juin 1855 (sur l'école anglaise).

23. - Louis Viau, décorateur de nombreuses églises de la région nantaise, ne laissa rien à la cathédrale (voir MAILLARD, É., op. cit., p. 141-142) mais réalisa un relevé des peintures du XVII siècle de la voûte du chœur roman avant sa destruction. Delaunay quant à lui œuvra surtout pour la Visitation et pour Saint-Nicolas.

24. - LACAMBRE, Geneviève et LACAMBRE, Jean. « La politique d'acquisition sous la Restauration : les tableaux d'histoire ». Bulletin de la Société de l'art français, 1972, p. 331-344, ici p. 339.

25. - Notre historique se fonde essentiellement sur DURVILLE, abbé Georges. Études sur le vieux Nantes d'après les documents originaux. Nantes: L. Durance, 1900-1915, p. CXXXCXXXI ; SÉRULLAZ, Maurice. Mémorial de l'exposition Eugène Delacroix organisée au musée du Louvre. Paris : Musées nationaux, 1963, no 6 ; GEORGEL, Pierre, ROSSI-BORTOLATTO, Luigina. Tout l'œuvre peint de Delacroix. Paris : Flammarion, 1975, p. 89, no 22 ; JOHNSON, Lee. The Paintings of Eugène Delacroix: a critical catalogue. Oxford: Clarendon press, 1981-1989. T. I, p. 163-165, no 153 ; Delacroix : peintures et dessins d'inspiration religieuse. Cat. expo., Nice, musée national du Message biblique Marc Chagall, juillet-octobre 1986. Paris : Réunion des musées nationaux, 1986, p. 44-45, no 2 ; SZEEMAN, Harald (dir.). Eugène Delacroix. Cat. expo., Zurich, Kunsthaus, Francfort, Städtische Galerie, 1987-1988, Zurich/Francfort: Kunsthaus/Städtische Galerie, 1987, p. 70-71; SÉRULLAZ, Maurice. Delacroix. Paris : Fayard, 1989, p.69-70; Géricault. Cat. expo. Paris, Grand Palais, 1991-1992. Paris: Réunion des musées nationaux, 1991, p. 287, 293, 296, 300-302, 320-321. 
26. - L'erreur provient de Villot ; elle est reprise par SPECTOR, Jack J.. « The Vierge du Sacré-Cœur : religious politics and personal expression in an early work of Delacroix ». The Burlington Magazine, vol. CXXIII, avril 1981, nº 937, p. 197-206.

27. - Par une lettre du 24 mars 1820 (conservée à la bibliothèque municipale de Nantes, ms. 2636, f. 2470), le ministère de l'Intérieur demandait des explications au préfet sur les dimensions du tableau, son emplacement, son éclairage... Un dessin de l'architecte Ogée fut envoyé à Paris le 23 avril 1820.

28. - L'une d'elles est conservée au musée Delacroix à Paris.

29. - Cité par le chanoine Durville, op. cit., p. CXXX.

30. - Bibl. mun. Nantes, ms. 2636, f. 2470.

31. - La cathédrale d'Ajaccio.

32. - Bibl. mun. Nantes, ms. 2636, f. 2470.

33. - Conservé à l'Art Institute de Chicago; voir ROSENBERG, Pierre. De David à Delacroix, la peinture française de 1774 à 1830. Cat. expo., Paris, Grand Palais, novembre 1974-février 1975. Paris : éd. des Musées nationaux, 1974, p. 148, n 47, pl. 84.

34. - Voir TAPIÉ, Alain, JOUBERT, Caroline. L'allégorie dans la peinture. La représentation de la charité au XVII ${ }^{e}$ siècle. Cat. expo., Caen, musée des Beaux-Arts, juin-octobre 1986. Caen : musée des BeauxArts, 1986, $\mathrm{n}^{\text {os }}$ 35-36. La chapelle de l'hôpital de Nantes en conservait autrefois une copie portant la mention «Ex. Dono. D. D. Christophori Ludovici Turpin de Crissé de Sanscay Episcopi Nantensis anno $1738 »$ avec les armoiries de l'évêque.

35. - DURVILLE, G., op. cit., p. CXXXI.

36. - FOUCART, B., op. cit, p. 261-262, fig. 224.

37. - Notre synthèse s'appuie sur les recherches de Mmes C. Cosneau (en 1984, archives diocésaines, carton 306) et O. Riffet (en 1991, dans tous les fonds nantais), sur l'ouvrage de B. Foucart, p. 88, et sur les notices du catalogue de l'exposition Flandrin. FOUCART, Jacques, FOUCART, Bruno. Hippolyte, Auguste et Paul Flandrin, une fraternité picturale au XIXe siècle. Cat. expo. Paris, musée du Luxembourg, novembre1984-février 1985, Lyon, musée des Beaux-Arts, marsmai 1985. Paris : Réunion des musées nationaux, 1984, p. 68-69, nos 11-12-13.

38. - LACAMBRE, G. et LACAMBRE, J., Art. cit. p. 331, n.1.

39. - Inv. 6538. Le Louvre en conserve également une esquisse (RF 1946-8). Sur le même thème, la Maclean Gallery de Londres présentait, il y a quelques années, une autre toile du même artiste des environs de 1822 représentant deux bustes (d'Homère et de Napoléon ?) et un entablement corinthien renversé.

40. - FLANDRIN, Hippolyte. Lettres et pensées d'Hippolyte Flandrin. Éd. Henri Delaborde. Paris : H. Plon, 1865, p. 239-240.

41. - GAUTIER, Théophile. Les Beaux-Arts en Europe : 1855. Paris : M. Lévy frères, 1855, p. 285.

42. - En définitive, Flandrin situa la scène à Rome, saint Clair ayant pu «avant de quitter la capitale du monde chrétien, opérer quelques-uns des miracles que la tradition lui attribue». Notice sur la chapelle Saint-Clair dans l'église cathédrale de Nantes. Nantes: impr. Vve Camille Mellinet, 1845 , p. 7.

43. - Et non pas un portrait de l'artiste et de son frère ; RUSSON, J.-B., DURET, D., op. cit., p. 105.

44. - Lettre à Lacuria du 24 mars 1836 citée par FLANDRIN, Louis. Un peintre chrétien au XIXe siècle. Hippolyte Flandrin. Paris : Perrin, 1909, p. 64-65, avec des variantes par rapport à FLANDRIN, H. éd. cit., p. 246.

45. - Vraisemblablement présentée au musée en 1842 ; voir Explication des ouvrages de peinture, sculpture, dessin et lithographie des artistes vivants exposés au Musée de Nantes le 5 juillet 1842. Nantes : Impr. Mellinet, 1842, p. 23, nº 139.

46. - Voir Explication des ouvrages de peinture, sculpture et dessin des artistes vivants exposés à la Bibliothèque de Nantes le 18 août 1854. Nantes : Impr. Mellinet, 1854, p. 22, $\mathrm{n}^{\text {os }} 186-188$. Laure était venu lui-même installer ses tableaux dans la chapelle Saint-Clair (Notice..., op. cit., p. 11). 
47. - Arch. dép. Loire-Atlantique, $125 \mathrm{~J}$ 306, lettre du 22 février 1845 de l'évêché à la préfecture: «le tableau accordé le 14 août 1844 par le ministre, de Jalabert représentant la Vierge visitant sainte Philomène, ne nous est point encore parvenu... ». Le sujet n'est donc pas exactement celui du tableau décrit dans la chapelle Saint-Clair en 1845 (Notice sur la chapelle Saint-Clair..., op. cit., p.7). En fait, Jean Jalabert (Carcassonne, 1815 - ?, 1901) reçut en 1844 un paiement de $800 \mathrm{~F}$ (Arch. nat., F21 0037) pour une copie de la Visitation de Sebastiano del Piombo (Paris, musée du Louvre), copie qui a disparu de Saint-Pierre à une date indéterminée. Il est à noter que l'homonyme et contemporain de Jean Jalabert, Charles-François Jalabert (Nîmes, 1819 - Paris, 1901), exposa parfois à Nantes.

48. - Voir FOUCART, B., op. cit., p. 231.

49. - Collection particulière, voir ibid., fig. 140.

50. - Villa romaine où travaillèrent Peter Von Cornelius (1783-1867) et Friedrich Overbeck (1789-1869) en 1817-1827.

51. - Le Wallraf-Richartz Museum de Cologne conserve entre autres des Crucifixions de ces deux maitres de la fin du XVe siècle.

52. - Voir Explication des ouvrages... exposés au Musée de Nantes le 5 juillet 1842, op. cit. p. 16, no 84 ; Explication des ouvrages de peinture, sculpture et dessin des artistes vivants exposés au Musée de Nantes le 1er août 1848. Op. cit., p. 12, no 61 ; Explication des ouvrages... exposés à la Bibliothèque de Nantes le 18 août 1854. Op. cit., p. 14, nos 107-108; Explication des ouvrages de peinture, sculpture, architecture des artistes vivants exposés dans le Muséum le 7 novembre 1872. Nantes: Impr. Mangin et Giraud, 1872, p. 25, no 228 ; European Nineteenth Century Watercolors, Drawings, Paintings and Sculpture. Cat. expo., hiver 1989-1990. New York : Shepherd Gallery, 1989-1990, no 32.

53. - Laïs de Corinthe, plâtre teinté, H. : $203 \mathrm{~cm}$, s.d. Doussault, 1868 ; Salon de 1869 ; don de l'auteur en 1873 ; cat. du Musée, 1876 ( $\left.\mathrm{n}^{\circ} 25\right), 1913$ ( $\left.\mathrm{n}^{\circ} 1780\right), 1953$ ( $\left.\mathrm{n}^{\circ} 1780\right)$.

54. - Proches de ceux de son père comme L.-R. des Courtils et son épouse, H/T : 161 x $134 \mathrm{~cm}$, s.d. 1808. Cat. Sotheby's Monaco, 2-3 décembre 1988, $\mathrm{n}^{\circ} 675$.

55. - Voir GRUNCHEC, Philippe, FOUCART-WALTER, Élisabeth. Le Grand prix de peinture. Les concours des prix de Rome de 1797 à 1863. Paris : École nationale supérieure des beaux-arts, 1983; notamment les années 1821 à 1826 ; BERNIER, Georges. Consulat, Empire, Restauration. Art in Early XIX Century France. Cat. expo., Londres, gal. Wildenstein, 1981, New York, Wildenstein and co, 1982, p. 74.

56. - DANJOU, Pierre-Nicolas. «Notice sur Charles-Auguste Van den Berghe... ». Mémoires de la Société académique d'archéologie, sciences et arts du département de l'oise, 1854, p. 345-376, décrit une Piéta et deux scènes de funérailles dont une nocturne. Le musée du Louvre a récemment recouvré sa Résurrection de Lazare (Salon de 1841) en dépôt à la cathédrale de Périgueux puis au musée de la ville (1910), dont la composition rappelle celle du tableau de Nantes; voir FOUCART, Jacques. Nouvelles acquisitions du Département des peintures, musée du Louvre (1987-1990). Paris : Réunion des musées nationaux, 1991, p. 214, repr. p. 215.

57. - DANJOU, P.-N., Art cit., p. 360.

58. - Atala au tombeau, Salon de 1808, H/T : 207 x 267 cm, Paris, musée du Louvre, Inv. 4958.

59. - Cité par Danjou, Art. cit., p. 360.

60. - Sur l'iconographie des deux saints, voir AUBERT, Roger, VAN CAUWENBERGH, Étienne. Dictionnaire d'histoire et de géographie ecclésiastique. Paris : Letouzey et Ané, 1960, tome 14, fasc. 78 à 83, p. 353 ; RUSSON, Jean-Baptiste. La Passion des enfants nantais. Nantes: Impr. de l'Éclaireur de l'Ouest, 1945, p. 124 (cite le tableau de Vauchelet); DURAND, Eugène. La Basilique Saint-Donatien, Nantes. s.l., 1986. 
61. - Arch. nat., $\mathrm{F}^{19} 7771$.

62. - La chapelle a brûlé en 1859. Une tête d'ange est conservée au musée de Châteauroux. Le décor est actuellement dissimulé par des dispositifs provisoires.

63. - Saint-Eustache abrite encore son Martyre de sainte Agnès (s.d. 1855), contrairement à ce qu'affirment certaines notices sur Vauchelet.

64. - Sur les rapports du peintre et de la duchesse de Berry, voir IVANOFF, Nicolas. «CharlesAchille d'Hardiviller, peintre de la duchesse de Berry ». Bulletin de la Société d'histoire de l'Art français, 1972, p. 345-348; RUSSCOL, Diane. « Le thème de la reine prisonnière : deux tableaux du Salon de 1833 ». La Revue du Louvre et des Musées de France, 1990, nº 2, p. 123-128.

65. - GABET, Charles. Dictionnaire des artistes de l'école française au XIX ${ }^{e}$ siècle. Paris: Mme Vergne, 1831.

66. - IVANOFF, N., Art. cit., p. 345, n.1 ; FOUCART, B., op. cit., p. 401.

67. - ROSSET, Anne-Marie. "Collection de Vinck, inventaire analytique » dans ROSSET, AnneMarie. Un siècle d'histoire de France par l'estampe, 1770-1871. T.V, La Restauration et les Cent-jours. Paris : Bibliothèque nationale, 1938, p. 574-586 ; ADHÉMAR, Jean, LETHÈVE, Jacques, GARDEY, Françoise. Inventaire du fonds français après 1800. Paris: Bibliothèque nationale, t. X, 1958, p. 121-124.

68. - Arch. nat., $\mathrm{F}^{19} 7771$.

69. - GUÉPIN, Ange, BONAMY, Eugène. Nantes au XIXe siècle, statistique topographique, industrielle et morale. Nantes: P. Sebire, 1835, rééd. Nantes: Centre de recherche politique, université de Nantes, 1981, p. 112.

70. - La place prépondérante de la Vierge dans la Descente de croix de Nantes, et la notice du catalogue du Salon de 1827 (MUSÉE DU LOUVRE. Explication des ouvrages... exposés au Musée Royal des Arts, le 4 novembre 1827. Paris : Impr. Vve Ballard, 1827, p. 78, $\mathrm{n}^{\circ} 433$ ) ne laissent aucun doute sur l'identification du tableau de Nantes : «La Sainte Vierge, restée près de la croix, est soutenue par une des saintes femmes, tandis que l'apôtre saint Jean et Joseph d'Arimathie portent au tombeau le corps du Christ. La Madeleine supporte un bras qu'elle arrose de ses larmes ».

71. - Bibl. mun. Nantes, ms. 2636, f. 2470.

72. - Voir BELLIER de LA CHAVIGNERIE, Émile, AUVRAY, Louis. Dictionnaire général des artistes de l'école française. Paris : Renouard, 1882-1885, 3 vol., t. I, p. 610 ; De David à Delacroix, op. cit, p. 423.

73. - Voir n.49.

74. - Le catalogue de l'exposition Madame, Duchesse de Berry (Nantes, musée Dobrée, décembre 1963- février 1964. Paris : Presses artistiques, 1963) ne mentionne rien de tel mais le fait pourrait éclairer de façon nouvelle la célèbre « amabilité de Mme la duchesse de Berri » dont Stendhal parle dans Lamiel.

75. - Le Portement de Croix du Louvre (Inv. 20525, h/t : 330 x $262 \mathrm{~cm}$, Salon de 1848) ne porte pas non plus de signature ; voir La Revue du Louvre et des Musées de France, 1978, nº 4, p. 305 ; FOUCART, B., op. cit., fig. 5.

76. - Ibid., p. 116.

77. - Garnier réalisa un Cortège nuptial de Napoléon et Marie-Louise (Versailles) et un dessus de porte pour les Tuileries : Les galeries du Louvre bâties par Henri IV (Versailles) que Jal critiqua également. Voir LACAMBRE, Geneviève et LACAMBRE, Jean. «La galerie de Diane aux Tuileries sous la Restauration ». La Revue du Louvre et des Musées de France, 1975, n 1, p. 39-50.

78. - Sur l'original de Le Sueur, voir MÉROT, Alain. Eustache Le Sueur, 1616-1655. Paris : Éd. Arthena, 1987, p. 302-305, fig. 413 et 413 bis.

79. - Arch. dép. Loire-Atlantique, $125 \mathrm{~J}$ 306, lettre du 20 juillet 1845 ; Arch. nat., $\mathrm{F}^{19} 7771$; FURET, J., CAILLÉ, D., op. cit., p. 230, n.1, signalent une esquisse du tableau dans la collection Joseph Chudeau ; s'agissait-il d'un modello soumis à l'approbation de l'évêque?

80. - Explication des ouvrages... exposés à la Bibliothèque de Nantes le 18 août 1854. 1854, p. 21, nº 169. 
81. - De Jolin, citons à Nantes : Saint Dominique ressuscitant un enfant (1846, à Saint-Nicolas), Saint Vincent Ferrier (1846, à Saint-Similien) et à Guérande : Le Christ et La Madeleine (1863, à la collégiale).

82. - En 1838, Donné et Sotta participent ensemble à l'exposition artistique d'Angers. Voir MAILLART, É., op. cit., p. 231-232.

83. - L'original d'Ottavio Vannini (Florence, 1585 - Id., 1644) fut peint en 1627 pour Laurent de Médicis. Saisi à Modène par les Français, il fut déposé à la cathédrale de 1809 à 1828, date à laquelle il intégra les collections du musée des Beaux-Arts. Voir BREJON de LAVERGNÉE, Arnauld. Peintures monumentales. Musée des beaux-arts de Nantes. Nantes : musée des Beaux-Arts, 1983, t. I, p. 32-34, $\mathrm{n}^{\circ}$ IV, et Seicento, le siècle de Caravage dans les collections françaises. Cat. expo., Paris, Grand Palais, octobre 1988-janvier 1989. Paris: Éd. de la Réunion des musées nationaux, 1988, p. 370-372, n 152 .

84. - Voir Explication des ouvrages de peinture, sculpture, architecture, gravure et lithographie des artistes vivants exposés dans la galerie des Beaux-Arts, Nantes, le samedi 10 août 1861. Nantes : 1861, Impr. Merson, p. 97, $\mathrm{n}^{\text {os }}$ 644-645 ; Explication des ouvrages de peinture, sculpture, architecture, gravure et lithographie des artistes vivants exposés au Palais du cours Saint-André. Nantes : 1886, Impr. du Commerce, $\mathrm{p} 53, \mathrm{n}^{\circ} 1748$.

85. - BAUDELAIRE, Charles. Écrits esthétiques. Paris: Union générale d'éditions, coll. «10/18», 1986, p. 151.

86. - L'original du Dominiquin est conservé à Versailles. Voir BREJON de LAVERGNÉE, Arnauld, VOLLE, Nathalie, MÉNÉGAUX, Odile. Répertoire des peintures italiennes du XVII siècle. Musées de France. Paris : Éd. de la Réunion des musées nationaux, 1988, p. 137.

87. - RUSSON, J.-B., DURET, D., op. cit., p. 108, la signalent dans le transept et en attribuent le modèle à Nicolas Poussin. Il s'agit en fait d'une copie de La Remise des clefs à saint Pierre peinte par Guido Reni (1575-1642) au début des années 1630 pour San Pietro in Valle à Fano (H/T : 2,10 x $3,42 \mathrm{~m}$ ). Commandée par le père Girolamo Gabrielli en même temps qu'une Annonciation (1621, in situ), la toile fut emportée à Paris pendant la période napoléonienne puis déposée au musée Rigaud de Perpignan de 1896 à 1986 ; elle a récemment réintégré les collections du Louvre. Voir BACCHESCHI, Edi. L'Opera completa di Guido Reni. Milan : Rizzoli, 1971, p. 100, n 104 ; Nell' età di Correggio e dei Carracci. Pittura in Emilia dei secoli XVI e XVII. Cat. expo. Bologne, Pinacoteca nazionale, septembre-novembre 1986, Washington, National Gallery of Art, décembre 1986-février 1987, New York, The Metropolitan Museum of Art, mars-mai 1987. Bologne/Washington/New York: Pinacoteca nazionale, Nuova Alfa editoriale/National Gallery of Art/The Metropolitan Museum of Art, 1986, p. 516-517, nº 184.

88. - Voir COSTA, Dominique. La Révolution à Nantes et la Vendée militaire. Cat. expo., Nantes, musée Dobrée, 1967. Nantes : musée Dobrée, $\mathrm{n}^{\circ} 244, \mathrm{pl} .40$.

89. - Voir Explications des ouvrages... exposés au Musée de Nantes le 5 juillet 1842. Op. cit., p. 31, $\mathrm{n}^{\circ} 211$. Cailliaud était conservateur du muséum d'Histoire naturelle.

90. - Voir Explication des ouvrages... exposés dans les galeries des Beaux-Arts, Nantes, le samedi 10 août 1861. 1861, $\mathrm{n}^{\circ} 592$.

91. - Voir Explication des ouvrages de peinture, sculpture, dessin et lithographie exposés au Musée de Nantes le 15 juillet 1845. Nantes: 1845, Impr. Mellinet, p. 36, $\mathrm{n}^{\circ} 245$. L'artiste avait obtenu une médaille pour ce portrait en 1842, à Angers.

92. - Voir Explication des ouvrages... exposés à la Bibliothèque de Nantes le 18 août 1854. 1854, p. 30, nº 277.

93. - Voir Explication des ouvrages de peinture, sculpture, dessin et lithographie exposés au Musée de Nantes le 15 avril 1858. Nantes : Impr. Mellinet, 1858, p. 33, nº 332. Aujourd'hui conservé au musée des Beaux-Arts (BENOIST, Luc. Ville de Nantes. Musée des beaux-arts. Catalogue et guide. Nantes : MBA, 1953, p. 190, n 1179, H/T : s.d. 1856). Le musée d'Orsay a acquis récemment un portrait d'Égidio Sotta par Delaunay. 
94. - RUSSON, J.-B., DURET, D., op. cit., p. 106. Ce programme répondait aux saints et aux anges dont Jules Laure avait décoré les boiseries de la chapelle Saint-Clair.

95. - Voir BELLI BARSALI, Isa. Mostra di Pompeo Batoni. Cat. expo., Lucca, 1967. Lucca : Azienda Grafica Lucchese, p. 61.

96. - JULIA, Isabelle, DUPONT, Patrick. Jules-Élie Delaunay. Cat. expo., Nantes, musée des BeauxArts, septembre-novembre 1988, Paris, musée Hébert, janvier-mars 1989. Saint-Sébastien : ACL Crocus, 1988, p. 145-165.

97. - Ibidem, p. 103-107, $\mathrm{n}^{\circ}$ 44. Sur la version du musée des Beaux-Arts, livrée fin septembre 1864 et achetée 4000 francs par l'État, la documentation des Peintures du musée d'Orsay conserve un intéressant dossier constitué par Mme Beauchamp. Voir également Arch. nat., $\mathrm{F}^{21} 132$.

98. - GUÉPIN, Ange. Essais historiques sur les progrès de la ville de Nantes. Nantes: P. Sebire, 1832, p. 282-283.

99. - PÉRON, André. Nantes et la Révolution : la mémoire des lieux. Quimper : Ressac, 1988, p. 37.

\section{RÉSUMÉS}

Dévastée par la Révolution, la vieille cathédrale romane de Nantes fut reconstruite sous la Restauration et son mobilier comme son décor entièrement renouvelés. Très peu de tableaux antérieurs au XIX ${ }^{\mathrm{e}}$ siècle y subsistent ; parmi les quelques rares toiles anciennes, la très maniériste Remise des clefs à saint Pierre de Charles Errard le père, jadis au maitre-autel, témoigne de ce que put être la peinture à Nantes au début $d u$ XvII ${ }^{\mathrm{e}}$ siècle (1619), tandis que le Saint Jean-Baptiste de François Lemoyne présente une facette plus convenue de l'art religieux à l'aube de la période rocaille (1715). La collection d'œuvres du XIX ${ }^{\mathrm{e}}$ siècle, elle, s'illustre par de grands noms d'artistes parisiens de l'époque romantique, ceux de Jean-Baptiste Mauzaisse, Hippolyte Flandrin, JeanBaptiste-François Désoria, Auguste Van den Berghe, Étienne-Barthélémy Garnier, même si Eugène Delacroix y brille par son absence... Mais les évêques et marguilliers de la cathédrale eurent aussi à cœur d'y faire travailler des Bretons ou des Nantais comme Vincent Vidal, Édouard Jolin, Joachim Sotta ou Jules-Élie Delaunay. Saint-Pierre de Nantes est donc devenu, comme bon nombre de lieux de culte de Paris et d'autres grandes villes françaises, un édifice fondamental pour étudier, comprendre et apprécier la grande peinture religieuse du XIX ${ }^{e}$ siècle.

Devastated during the French Revolution, the old Romanesque cathedral of Nantes was rebuilt during the period of the Restoration from 1815 to 1830. Its furnishings, like its decoration, was entirely renewed. Very few paintings dating from earlier than the nineteenth century survive, although, amongst these rare early survivors, the highly mannerist Remise des clefs à saint Pierre (Giving the keys to Saint Peter) by Charles Errard the elder, formerly at the main altar, shows what painting was like at Nantes at the beginning of the seventeenth century (1619). The painting of Saint John the Baptist by François Lemoyne offers a more conventional example of religious art at the beginning of the Rocaille period (1715). As for the collection of nineteenthcentury paintings, it is characterized by the names of the leading Parisian painters of the Romantic period, such as Jean-Baptiste Mauzaisse, Hippolyte Flandrin, Jean-Baptiste-François Désoria, Auguste Van den Berghe and Étienne-Barthélémy Garnier. Eugène Delacroix, however, is remarkable for his absence! But the bishops and wardens of the cathedral were also anxious to commission works from local Nantes and Breton painters such as Vincent Vidal, Édouard Jolin, Joachim Sotta or Jules-Élie Delaunay. The Saint-Pierre de Nantes cathedral, like other places of 
worship in Paris or in other major cities, has become a fundamental place for studying, understanding and appreciating the religious painting of the nineteenth century.

\section{INDEX}

Mots-clés : Nantes, cathédrale, Errard (Charles), Lemoyne (François), Mauzaisse (Jean-Baptiste), Flandrin (Hippolyte), Désoria (Jean-Baptiste-François), Van den Berghe (Auguste), Garnier (Étienne-Barthélémy), Delacroix (Eugène), Vidal (Vincent), Jolin (Édouard), Sotta (Joachim), Delaunay (Jules-Élie)

Keywords : Nantes, cathedral, Errard (Charles), Lemoyne (François), Mauzaisse (Jean-Baptiste), Flandrin (Hippolyte), Désoria (Jean-Baptiste-François), Van den Berghe (Auguste), Garnier (Étienne-Barthélémy), Delacroix (Eugène), Vidal (Vincent), Jolin (Édouard), Sotta (Joachim), Delaunay (Jules-Élie)

\section{AUTEUR}

\section{PIERRE CURIE}

Département restauration, Centre de recherche et de restauration des musées de France pierre.curie@culture.gouv.fr 